\title{
Understanding the mechanism of the divergent reactivity of non-heteroatom-stabilized chromium carbene complexes with furfural imines: formation of benzofurans and azetines
}

Ignacio Funes-Ardoiz, ${ }^{[\mathrm{a}]}$ Jairo González, ${ }^{[\mathrm{b}]}$ Javier Santamaría* ${ }^{[\mathrm{b}]}$ and Diego Sampedro* ${ }^{[\mathrm{a}]}$

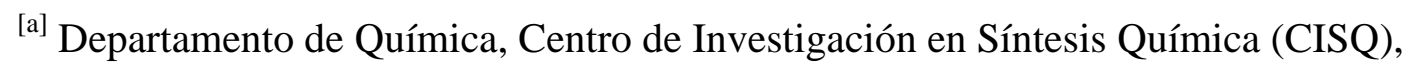
Universidad de La Rioja, C/ Madre de Dios, 53, 26006, Logroño (Spain).

[b] Departamento de Química Orgánica e Inorgánica e Instituto Universitario de Química Organometálica "Enrique Moles", Unidad Asociada al C.S.I.C. Universidad de Oviedo, C/ Julián Clavería, 8, 33006 Oviedo, Spain E-mail: diego.sampedro@unirioja.es

\section{Table of Contents}

1. Attack of the C-3 in Furan Moiety to the Carbene Carbon ................................... 2

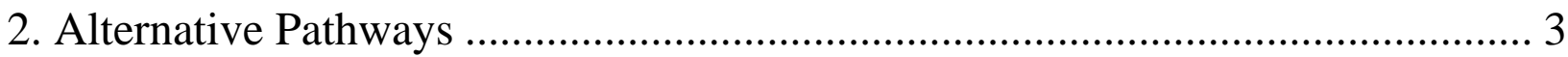

3. Computed Energies ................................................................................................. 4

4. Cartesian coordinates and calculated potential energies (atomic units) of the

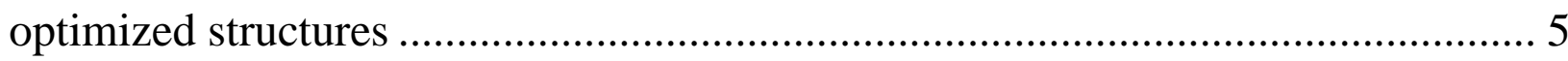

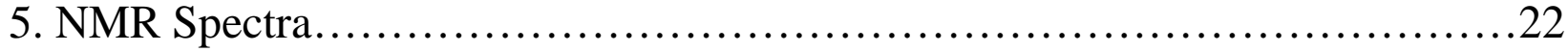



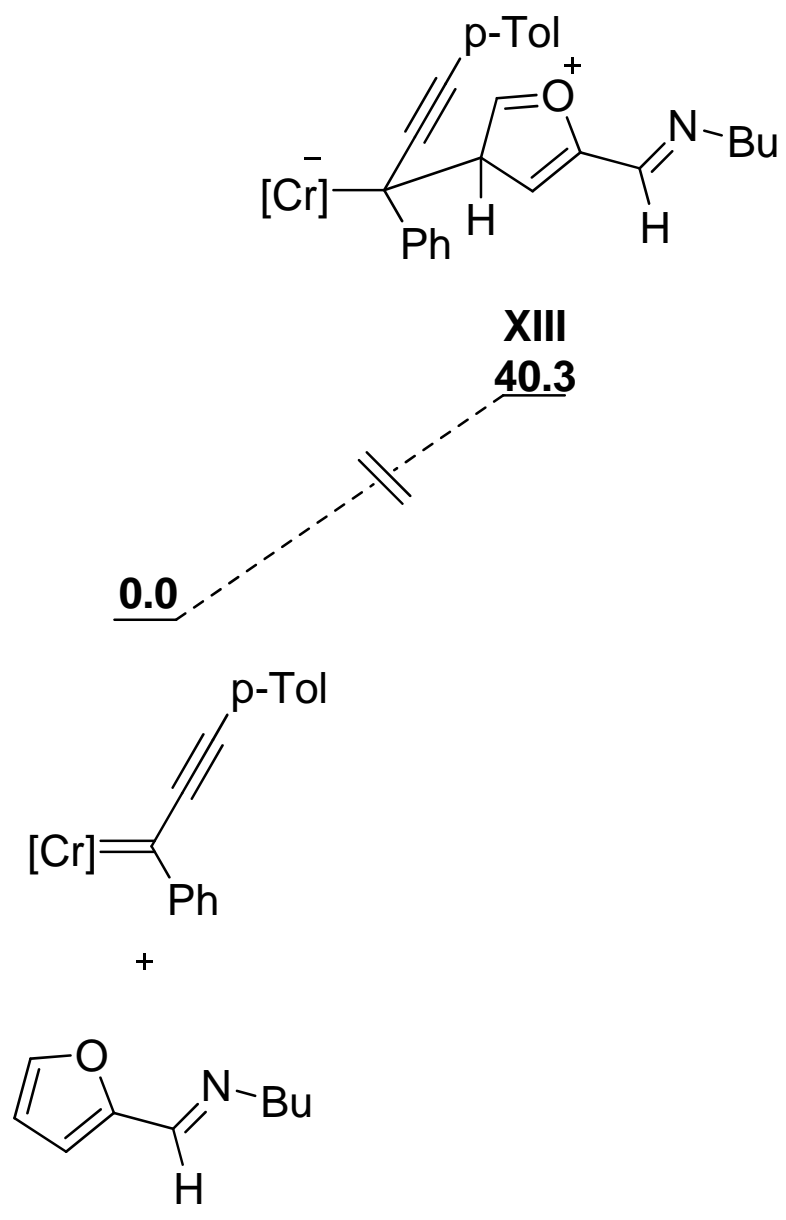

Figure S1. Attack to the carbenic carbon by the $\mathrm{C}-3$ in the furan moiety. 


\section{Alternative Pathways}

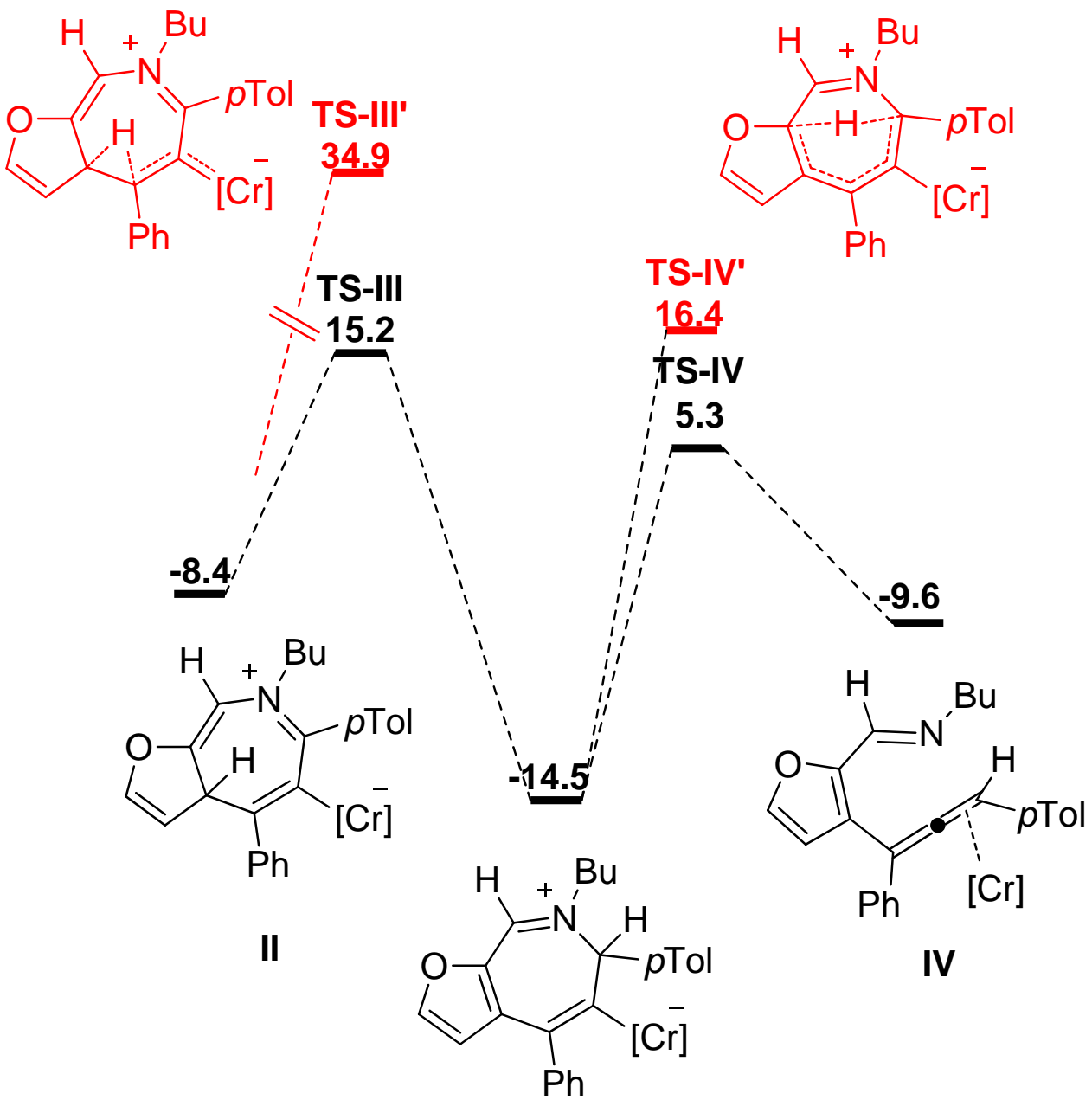

III

Figure S2. Alternative pathways from intermediate II.

We also explored the possibilities of a [1,3] hydrogen migration from intermediate II. Unfortunately, all our attempts ended always in the initial intermediate. Alternatively, a [1,2] hydrogen migration was found to be very high in terms of free energy $(34.9 \mathrm{kcal} / \mathrm{mol})$. In addition, we also explored a sequence of $[1,5]$ hydrogen migrations in order to protonate the metal-carbon bond. The first of these migrations resulted higher in energy than the original mechanism. Thus, this alternative pathway seems to be not competitive. 


\section{Computed Energies}

\begin{tabular}{|c|c|c|c|c|c|c|c|}
\hline Name & SCF & G correction & total low & SCF high & total high & Relative Low & Relative High \\
\hline $3 b$ & -1269.179682 & 0.206703 & -1268.972979 & -1269.464421 & -1269.257718 & 0.0 & 0.0 \\
\hline 4 & -480.401429 & 0.167139 & -480.234289 & -480.517777 & -480.350638 & & \\
\hline I & -1749.624834 & 0.401932 & -1749.222902 & -1750.023918 & -1749.621986 & -9.8 & -8.6 \\
\hline II & -1749.631160 & 0.408244 & -1749.222916 & -1750.03006 & -1749.621816 & -9.8 & -8.4 \\
\hline III & -1749.646729 & 0.413007 & -1749.233722 & -1750.044416 & -1749.631409 & -16.6 & -14.5 \\
\hline IV & -1749.628757 & 0.406819 & -1749.221938 & -1750.030465 & -1749.623646 & -9.2 & -9.6 \\
\hline V & -1749.628995 & 0.40487 & -1749.224125 & -1750.030143 & -1749.625273 & -10.6 & -10.6 \\
\hline VI & -1749.632199 & 0.408818 & -1749.223381 & -1750.034837 & -1749.626019 & -10.1 & -11.1 \\
\hline VII & -1749.615512 & 0.403675 & -1749.211838 & -1750.019296 & -1749.615621 & -2.9 & -4.6 \\
\hline VIII & -1749.679098 & 0.409528 & -1749.26957 & -1750.078641 & -1749.669113 & -39.1 & -38.1 \\
\hline $5 b-C r$ & -1749.713575 & 0.407473 & -1749.306102 & -1750.114583 & -1749.70711 & -62.0 & -62.0 \\
\hline IX & -1749.594019 & 0.407483 & -1749.186537 & -1749.996274 & -1749.588791 & 13.0 & 12.3 \\
\hline$x$ & -1749.623111 & 0.404202 & -1749.218909 & -1750.022492 & -1749.61829 & -7.3 & -6.2 \\
\hline $\mathrm{XI}$ & -1749.630030 & 0.409121 & -1749.22091 & -1750.029004 & -1749.619883 & -8.6 & -7.2 \\
\hline XII & -1749.646381 & 0.413136 & -1749.233245 & -1750.044116 & -1749.63098 & -16.3 & -14.2 \\
\hline XIII & -1749.543501 & 0.401481 & -1749.142021 & -1749.945664 & -1749.544183 & 40.9 & 40.3 \\
\hline $6 b$ & -1749.640835 & 0.4048 & -1749.236035 & -1750.038435 & -1749.633635 & -18.1 & -15.9 \\
\hline TS-I & -1749.578730 & 0.404138 & -1749.174592 & -1749.980439 & -1749.576301 & 20.5 & 20.1 \\
\hline TS-II & -1749.592139 & 0.403176 & -1749.188964 & -1749.993174 & -1749.589998 & 11.5 & 11.5 \\
\hline TS-III & -1749.592440 & 0.406462 & -1749.185978 & -1749.990537 & -1749.584075 & 13.4 & 15.2 \\
\hline TS-IV & -1749.611092 & 0.408978 & -1749.202114 & -1750.008958 & -1749.59998 & 3.2 & 5.3 \\
\hline TS-V & -1749.623606 & 0.406278 & -1749.217328 & -1750.024639 & -1749.618361 & -6.3 & -6.3 \\
\hline TS-VI & -1749.585699 & 0.40161 & -1749.184089 & -1749.986856 & -1749.585246 & 14.5 & 14.5 \\
\hline TS-VIII & -1749.604035 & 0.403394 & -1749.20064 & -1750.005533 & -1749.602139 & 4.2 & 3.9 \\
\hline TS-IX & -1749.574309 & 0.405595 & -1749.168713 & -1749.976638 & -1749.571043 & 24.2 & 23.4 \\
\hline TS-X & -1749.587722 & 0.404335 & -1749.183387 & -1749.991284 & -1749.586949 & 15.0 & 13.4 \\
\hline TS-XI & -1749.592323 & 0.403911 & -1749.188412 & -1749.993208 & -1749.589297 & 11.8 & 12.0 \\
\hline TS-XII & -1749.591639 & 0.406321 & -1749.185317 & -1749.989672 & -1749.583351 & 13.8 & 15.7 \\
\hline TS-aze & -1749.585272 & 0.403309 & -1749.181963 & -1749.98343 & -1749.580121 & 15.9 & 17.7 \\
\hline TS-III' & -1749.557501 & 0.403637 & -1749.153864 & -1749.956458 & -1749.552821 & 33.5 & 34.8 \\
\hline TS-IV' & -1749.590572 & 0.406317 & -1749.184255 & -1749.988601 & -1749.582284 & 14.4 & 16.4 \\
\hline
\end{tabular}




\section{Cartesian coordinates and calculated potential energies (atomic units) of}

the optimized structures

$\begin{array}{lrrr}\text { 3b } & & & \\ & & & \\ \text { E= -1269.179682H } & & \\ \text { Cr } & -1.91732500 & 0.00130500 & -0.17223100 \\ \text { C } & -1.70996800 & 1.66947500 & -1.05430600 \\ \text { C } & -2.17557000 & 0.90513600 & 1.47370600 \\ \text { C } & -3.77157600 & 0.05003000 & -0.44658300 \\ \text { C } & -2.06821000 & -1.70969800 & 0.62956200 \\ \text { C } & -1.68127700 & -0.86067100 & -1.84529900 \\ \mathrm{O} & -2.17578700 & -2.76647900 & 1.08239900 \\ \mathrm{O} & -2.37431200 & 1.47648800 & 2.45721400 \\ \mathrm{O} & -1.57737400 & 2.66850100 & -1.61584900 \\ \mathrm{O} & -1.56248500 & -1.37809500 & -2.86980600 \\ \mathrm{C} & 0.13889200 & -0.01563800 & 0.12943300 \\ \mathrm{C} & 0.91833300 & -0.23211000 & 1.34699100 \\ \mathrm{C} & 2.30793900 & 0.02819000 & 1.37028500 \\ \mathrm{C} & 0.32528500 & -0.69437700 & 2.53916900 \\ \mathrm{C} & 3.05615600 & -0.15041300 & 2.52283800 \\ \mathrm{H} & 2.79691500 & 0.39071200 & 0.46686400 \\ \mathrm{C} & 1.07555700 & -0.89068000 & 3.68864600 \\ \mathrm{H} & -0.73839200 & -0.91455100 & 2.56622500 \\ \mathrm{C} & 2.44236800 & -0.61407700 & 3.68678000 \\ \mathrm{H} & 4.12215800 & 0.07039900 & 2.51641500 \\ \mathrm{H} & 0.59272400 & -1.25765500 & 4.59250300 \\ \mathrm{H} & 3.02841300 & -0.76028600 & 4.59280300 \\ \mathrm{C} & 0.87327000 & 0.25316700 & -1.01284500 \\ \mathrm{C} & 2.07878600 & 0.74260300 & -3.31025300 \\ \mathrm{C} & 1.30915400 & 1.07388600 & -4.44364700 \\ \mathrm{C} & 3.48028500 & 0.66969400 & -3.43412800 \\ \mathrm{C} & 1.92784300 & 1.32107600 & -5.65709900 \\ \mathrm{H} & 0.22471400 & 1.13168800 & -4.35151800 \\ \mathrm{C} & 4.08451400 & 0.91248500 & -4.65599700 \\ \mathrm{H} & 4.07785700 & 0.41221400 & -2.56034700 \\ \mathrm{C} & 3.32211700 & 1.24286100 & -5.78514000 \\ \mathrm{H} & 1.32606000 & 1.57464500 & -6.53020200 \\ \mathrm{H} & 5.16886700 & 0.84560100 & -4.74621100 \\ \mathrm{O} & -4.91909800 & 0.08244600 & -0.60253300 \\ \mathrm{C} & 1.44949400 & 0.48047000 & -2.07590300 \\ \mathrm{C} & 3.98307500 & 1.52814400 & -7.09510400 \\ \mathrm{H} & 4.19084300 & 2.60338700 & -7.19932900 \\ \mathrm{H} & 4.94119700 & 1.00210100 & -7.18706500 \\ \mathrm{H} & 3.34505700 & 1.24082800 & -7.94022400 \\ & & & \\ & & & \end{array}$

4

$\begin{array}{lrrr}\mathrm{E}=-480.401429 \mathrm{H} & & \\ \mathrm{N} & 2.56392700 & 3.47144900 & -2.16497200 \\ \mathrm{C} & 1.71197200 & 4.14585700 & -1.49887400 \\ \mathrm{H} & 1.05903600 & 4.91710800 & -1.93650300 \\ \mathrm{C} & 2.72513600 & 3.69677800 & -3.58959000 \\ \mathrm{H} & 3.79377000 & 3.91642400 & -3.75209600 \\ \mathrm{H} & 2.56228400 & 2.72278900 & -4.08118900 \\ \mathrm{C} & 1.88104000 & 4.75799000 & -4.27468800\end{array}$

$\begin{array}{rrr}0.80852800 & 4.53086000 & -4.15219500 \\ 2.04791800 & 5.74132400 & -3.80302800 \\ 2.20816100 & 4.85324300 & -5.75881300 \\ 3.28007600 & 5.07897700 & -5.88085600 \\ 2.04811200 & 3.87066100 & -6.23212400 \\ 1.37808200 & 5.90737200 & -6.47136300 \\ 1.53837800 & 6.90283600 & -6.03206300 \\ 1.62920600 & 5.97087600 & -7.53853000 \\ 0.30366500 & 5.68432500 & -6.39679800 \\ 1.53464700 & 3.94091000 & -0.07608700 \\ 2.10759500 & 3.11287300 & 0.84929100 \\ 0.59602100 & 4.72813600 & 0.53003200 \\ 1.48521900 & 3.40848400 & 2.09449300 \\ 2.88245700 & 2.38106000 & 0.65092900 \\ 0.57933800 & 4.39396900 & 1.83996700 \\ 1.68138200 & 2.94897300 & 3.05636300 \\ -0.12109400 & 4.93953900 & 2.46006900\end{array}$

TS I 


$\begin{array}{crrr}\mathrm{O} & -3.56469700 & 1.16834600 & -5.29532400 \\ \mathrm{C} & 2.22990100 & 1.13737100 & -1.97932100 \\ \mathrm{~N} & 2.40577200 & 3.26691300 & -2.25667700 \\ \mathrm{C} & 1.78820500 & 4.09730100 & -1.50631000 \\ \mathrm{H} & 1.40463200 & 5.05455000 & -1.88768000 \\ \mathrm{C} & 2.71078200 & 3.57129000 & -3.64598400 \\ \mathrm{H} & 3.78184800 & 3.83294300 & -3.68999800 \\ \mathrm{H} & 2.61592900 & 2.62742000 & -4.20369500 \\ \mathrm{C} & 1.87957800 & 4.64445900 & -4.32839300 \\ \mathrm{H} & 0.80707900 & 4.39881900 & -4.22569600 \\ \mathrm{H} & 2.02777900 & 5.62422100 & -3.84609800 \\ \mathrm{C} & 2.23872400 & 4.76299700 & -5.80331600 \\ \mathrm{H} & 3.31034700 & 5.00251800 & -5.89775800 \\ \mathrm{H} & 2.09872900 & 3.78574800 & -6.29276400 \\ \mathrm{C} & 1.40951400 & 5.81803500 & -6.51519200 \\ \mathrm{H} & 1.54963800 & 6.80951300 & -6.06031300 \\ \mathrm{H} & 1.67925800 & 5.89801000 & -7.57651200 \\ \mathrm{H} & 0.33712100 & 5.57980200 & -6.46324100 \\ \mathrm{C} & 1.55632500 & 3.87740800 & -0.10284200 \\ \mathrm{C} & 2.04599500 & 3.00950200 & 0.83837900 \\ \mathrm{O} & 0.72711700 & 4.78520700 & 0.50111800 \\ \mathrm{C} & 1.48718700 & 3.40377200 & 2.08125900 \\ \mathrm{H} & 2.72943400 & 2.18846200 & 0.65084000 \\ \mathrm{C} & 0.69017500 & 4.47868400 & 1.81203000 \\ \mathrm{H} & 1.65066100 & 2.95032400 & 3.05190500 \\ \mathrm{H} & 0.06128300 & 5.10733600 & 2.43074300 \\ \mathrm{C} & 7.35060700 & -0.79679500 & -3.73287100 \\ \mathrm{H} & 7.24597000 & -1.63432500 & -4.43307200 \\ \mathrm{H} & 7.93018500 & -0.00969600 & -4.23577200 \\ \mathrm{H} & 7.95429600 & -1.13866300 & -2.88085700\end{array}$

\begin{tabular}{lrrr}
$\mathrm{C}$ & 4.40952000 & 2.00604900 & -2.66193900 \\
$\mathrm{C}$ & 4.42896400 & -0.76389100 & -2.78380600 \\
$\mathrm{H}$ & 2.29293800 & -0.64670800 & -2.55882800 \\
$\mathrm{C}$ & 5.61032900 & 1.31730100 & -2.82682900 \\
$\mathrm{H}$ & 4.42499600 & 3.09634700 & -2.61319200 \\
$\mathrm{C}$ & 5.64606400 & -0.07557500 & -2.88947100 \\
$\mathrm{H}$ & 4.42672500 & -1.85399300 & -2.83764700 \\
$\mathrm{H}$ & 6.54206000 & 1.87965100 & -2.90571500 \\
$\mathrm{O}$ & -3.60605500 & -0.88154800 & -5.20393700 \\
$\mathrm{C}$ & 1.91890400 & 2.00840000 & -2.35255700 \\
$\mathrm{~N}$ & 1.91553800 & 3.44874300 & -2.58502300 \\
$\mathrm{C}$ & 1.93692900 & 4.29907200 & -1.60096900 \\
$\mathrm{H}$ & 1.86855600 & 5.35770100 & -1.85492600 \\
$\mathrm{C}$ & 1.77371500 & 3.85153800 & -4.00026800 \\
$\mathrm{H}$ & 2.48707200 & 3.22911300 & -4.55945200 \\
$\mathrm{H}$ & 0.76466000 & 3.53158600 & -4.30518800 \\
$\mathrm{C}$ & 2.00281500 & 5.31549200 & -4.29624600 \\
$\mathrm{H}$ & 1.23565300 & 5.93733700 & -3.80762700 \\
$\mathrm{H}$ & 2.98172600 & 5.63065300 & -3.89647400 \\
$\mathrm{C}$ & 1.95957200 & 5.57233800 & -5.79800900 \\
$\mathrm{H}$ & 2.73458900 & 4.96731400 & -6.29371200 \\
$\mathrm{H}$ & 0.99407800 & 5.22518400 & -6.19856500 \\
$\mathrm{C}$ & 2.15839300 & 7.04136300 & -6.12739300 \\
$\mathrm{H}$ & 3.12935600 & 7.40394300 & -5.75999300 \\
$\mathrm{H}$ & 2.12575200 & 7.21972600 & -7.20982200 \\
$\mathrm{H}$ & 1.37694400 & 7.66074000 & -5.66433700 \\
$\mathrm{C}$ & 2.03585000 & 4.01467200 & -0.21730700 \\
$\mathrm{C}$ & 2.17455700 & 2.90816100 & 0.59984500 \\
$\mathrm{O}$ & 1.99176200 & 5.14556000 & 0.56978900 \\
$\mathrm{C}$ & 2.21083600 & 3.38422500 & 1.92470700 \\
$\mathrm{H}$ & 2.24538000 & 1.87535500 & 0.28008300 \\
$\mathrm{C}$ & 2.09172900 & 4.74609000 & 1.84104300 \\
$\mathrm{H}$ & 2.29924900 & 2.79542000 & 2.82950200 \\
$\mathrm{H}$ & 2.06044100 & 5.52793800 & 2.59067100 \\
$\mathrm{C}$ & 6.93197100 & -0.82143100 & -3.07572600 \\
$\mathrm{H}$ & 7.09122200 & -1.55837500 & -2.27605500 \\
$\mathrm{H}$ & 6.93961100 & -1.37778900 & -4.02393400 \\
$\mathrm{H}$ & 7.79323100 & -0.14179100 & -3.08237500 \\
& & & \\
\hline
\end{tabular}

$\begin{array}{lrrr}\mathrm{E}=-1749.624834 \mathrm{H} & & \\ \mathrm{Cr} & -1.69738600 & 0.16336500 & -3.12910000 \\ \mathrm{C} & -3.07106600 & -0.08911200 & -1.85659600 \\ \mathrm{C} & -0.98819500 & -1.53036200 & -2.68980000 \\ \mathrm{C} & -2.86401400 & -0.47944700 & -4.39884500 \\ \mathrm{C} & -0.38966600 & 0.32561700 & -4.48293500 \\ \mathrm{C} & -2.18935400 & 1.97093600 & -3.34923500 \\ \mathrm{O} & 0.36912000 & 0.38532300 & -5.35633300 \\ \mathrm{O} & -0.54014000 & -2.56243400 & -2.41700200 \\ \mathrm{O} & -3.95126200 & -0.28191000 & -1.13015600 \\ \mathrm{O} & -2.45609600 & 3.09339100 & -3.45202900 \\ \mathrm{C} & -0.35284700 & 0.93781100 & -1.60611400 \\ \mathrm{C} & -0.67691500 & 0.85305100 & -0.16464400 \\ \mathrm{C} & -1.53082700 & 1.79284500 & 0.42648100 \\ \mathrm{C} & -0.13030200 & -0.15925600 & 0.63342100 \\ \mathrm{C} & -1.82116100 & 1.72752500 & 1.78594900 \\ \mathrm{H} & -1.96643900 & 2.58149500 & -0.19024100 \\ \mathrm{C} & -0.42590600 & -0.22424700 & 1.99384400 \\ \mathrm{H} & 0.54178900 & -0.89220400 & 0.18284200 \\ \mathrm{C} & -1.27093900 & 0.71845900 & 2.57654300 \\ \mathrm{H} & -2.48410500 & 2.46964600 & 2.23022400 \\ \mathrm{H} & 0.01033500 & -1.01698900 & 2.60110500 \\ \mathrm{H} & -1.50354800 & 0.66559300 & 3.63931800 \\ \mathrm{C} & 0.76967000 & 1.46390700 & -1.97670000 \\ \mathrm{C} & 3.19725400 & 1.31450000 & -2.55490500 \\ \mathrm{C} & 3.22945200 & -0.08840000 & -2.62307200\end{array}$

\section{TS II}

$\begin{array}{lrrr}\mathrm{E}=-1749.592139 \mathrm{H} & & \\ \mathrm{Cr} & -0.83665700 & 0.30082500 & -2.86792800 \\ \mathrm{C} & -2.17674500 & 0.09101800 & -1.54051500 \\ \mathrm{C} & -0.17107800 & -1.40326300 & -2.35383200 \\ \mathrm{C} & -2.09068900 & -0.41932200 & -3.96902600 \\ \mathrm{C} & 0.20624500 & 0.34757400 & -4.45402100 \\ \mathrm{C} & -1.44731700 & 2.07564000 & -3.15717100 \\ \mathrm{O} & 0.69629900 & 0.31884000 & -5.49997100 \\ \mathrm{O} & 0.19547900 & -2.44426000 & -2.01652600 \\ \mathrm{O} & -3.10271300 & -0.13173200 & -0.88588200 \\ \mathrm{O} & -1.84157900 & 3.14954600 & -3.31145700 \\ \mathrm{C} & 0.48590500 & 1.31492100 & -0.34780500 \\ \mathrm{C} & -0.37321300 & 0.95839700 & 0.73280000 \\ \mathrm{C} & -1.35456000 & 1.86312700 & 1.17709000 \\ \mathrm{C} & -0.22575100 & -0.27531700 & 1.39050800 \\ \mathrm{C} & -2.17096400 & 1.53364100 & 2.25177300 \\ \mathrm{H} & -1.48706000 & 2.81058000 & 0.65273000 \\ \mathrm{C} & -1.05458300 & -0.59897000 & 2.45744600 \\ \mathrm{H} & 0.53953000 & -0.97252500 & 1.04911400\end{array}$




$\begin{array}{rrrr}\mathrm{C} & -2.02598200 & 0.30377100 & 2.89305300 \\ \mathrm{H} & -2.93211900 & 2.23712900 & 2.58650700 \\ \mathrm{H} & -0.94160100 & -1.56182200 & 2.95389000 \\ \mathrm{H} & -2.66948400 & 0.04703500 & 3.73320100 \\ \mathrm{C} & 0.91238300 & 1.34847400 & -1.54083900 \\ \mathrm{C} & 2.96348600 & 1.36272300 & -2.98900500 \\ \mathrm{C} & 3.06362900 & -0.03889100 & -3.05372500 \\ \mathrm{C} & 4.08907800 & 2.10482700 & -3.38719600 \\ \mathrm{C} & 4.21190800 & -0.65840300 & -3.52112500 \\ \mathrm{H} & 2.22044900 & -0.65228600 & -2.74157400 \\ \mathrm{C} & 5.23369300 & 1.47594700 & -3.86407300 \\ \mathrm{H} & 4.09190000 & 3.18909500 & -3.28581800 \\ \mathrm{C} & 5.31975200 & 0.08458200 & -3.94820900 \\ \mathrm{H} & 4.25121000 & -1.74821200 & -3.56178000 \\ \mathrm{H} & 6.09037600 & 2.08348800 & -4.15960900 \\ \mathrm{O} & -2.88679400 & -0.88102800 & -4.68302400 \\ \mathrm{C} & 1.78457500 & 2.01866400 & -2.42537500 \\ \mathrm{~N} & 1.53402600 & 3.35365300 & -2.67672600 \\ \mathrm{C} & 0.99528700 & 4.18237800 & -1.74071700 \\ \mathrm{H} & 0.42777300 & 5.03526000 & -2.11033300 \\ \mathrm{C} & 1.61802700 & 3.88793600 & -4.05707100 \\ \mathrm{H} & 2.08775000 & 3.11484200 & -4.67389900 \\ \mathrm{H} & 0.58434100 & 4.00469600 & -4.42077900 \\ \mathrm{C} & 2.37110300 & 5.19994500 & -4.17521100 \\ \mathrm{H} & 1.79511400 & 6.01601900 & -3.71196300 \\ \mathrm{H} & 3.32118100 & 5.13943300 & -3.61933000 \\ \mathrm{C} & 2.65099900 & 5.53743900 & -5.63291000 \\ \mathrm{H} & 3.27113700 & 4.73950300 & -6.07378700 \\ \mathrm{H} & 1.70480900 & 5.53752300 & -6.19802700 \\ \mathrm{C} & 3.34620500 & 6.87881000 & -5.78804100 \\ \mathrm{H} & 4.29834900 & 6.89624000 & -5.23799300 \\ \mathrm{H} & 3.56555100 & 7.10419100 & -6.83979700 \\ \mathrm{H} & 2.72399700 & 7.69573400 & -5.39527900 \\ \mathrm{C} & 1.10720200 & 3.99380400 & -0.37405500 \\ \mathrm{C} & 1.79827600 & 3.07035700 & 0.43872700 \\ \mathrm{O} & 0.39296000 & 4.85069000 & 0.43482100 \\ \mathrm{C} & 1.54546900 & 3.47441900 & 1.78469100 \\ \mathrm{H} & 2.61515300 & 2.43915300 & 0.10665000 \\ \mathrm{C} & 0.67739400 & 4.51426300 & 1.71981400 \\ \mathrm{H} & 1.93196400 & 3.01671600 & 2.68791000 \\ \mathrm{H} & 0.18592600 & 5.11090900 & 2.47897900 \\ \mathrm{C} & 6.54851900 & -0.59713000 & -4.46556500 \\ \mathrm{H} & 6.93362100 & -1.33396700 & -3.74688900 \\ \mathrm{H} & 6.34178300 & -1.14238300 & -5.39765600 \\ \mathrm{H} & 7.35030000 & 0.12264700 & -4.67184400 \\ & & & \end{array}$

II

$\begin{array}{lrrr}\mathrm{E}=-1749.631160 \mathrm{H} & & \\ \mathrm{Cr} & -0.87649000 & 0.39188900 & -2.81425400 \\ \mathrm{C} & -2.15136400 & 0.29492200 & -1.40892400 \\ \mathrm{C} & -0.23023700 & -1.30077700 & -2.25821300 \\ \mathrm{C} & -2.20573400 & -0.38588500 & -3.80578800 \\ \mathrm{C} & 0.07247000 & 0.37347500 & -4.45038700 \\ \mathrm{C} & -1.55793700 & 2.11627300 & -3.19938600 \\ \mathrm{O} & 0.51067800 & 0.31120000 & -5.52101600 \\ \mathrm{O} & 0.10657700 & -2.34964200 & -1.90841500 \\ \mathrm{O} & -3.06711700 & 0.17213000 & -0.71521000 \\ \mathrm{O} & -2.04557700 & 3.13706400 & -3.43794500 \\ \mathrm{C} & 0.62135900 & 1.76310000 & -0.22809500\end{array}$

$\begin{array}{lll}-0.20146300 & 1.10059700 & 0.80535200\end{array}$

$\begin{array}{lll}-1.20258800 & 1.78082700 & 1.51121300\end{array}$

$\begin{array}{lll}0.03990200 & -0.24361700 & 1.11539900\end{array}$

$\begin{array}{lll}-1.94180500 & 1.13230100 & 2.49552100\end{array}$

$\begin{array}{lll}-1.42670100 & 2.81962400 & 1.26647000\end{array}$

$\begin{array}{lll}-0.69956100 & -0.89436800 & 2.09992800\end{array}$

$\begin{array}{lll}0.82760300 & -0.77492800 & 0.57985300\end{array}$

$\begin{array}{lll}-1.69116800 & -0.20597900 & 2.79552300\end{array}$

$\begin{array}{lll}-2.72598000 & 1.67225200 & 3.02522000\end{array}$

$\begin{array}{lll}-0.49530400 & -1.94033100 & 2.32649100\end{array}$

$-2.26875700 \quad-0.71113500 \quad 3.56870500$

$\begin{array}{llll}0.68377200 & 1.42300500 & -1.52348900\end{array}$

$\begin{array}{llll}2.75753500 & 1.40490400 & -2.95184100\end{array}$

$\begin{array}{lll}2.85882300 & 0.00936900 & -3.04702700\end{array}$

$\begin{array}{lll}3.92866900 & 2.15376500 & -3.16205000\end{array}$

$\begin{array}{lll}4.05581900 & -0.60363300 & -3.38307500\end{array}$

$\begin{array}{lll}1.98177700 & -0.60384300 & -2.86604100\end{array}$

$\begin{array}{llll}5.12536900 & 1.53497500 & -3.49794000\end{array}$

$\begin{array}{lll}3.92054300 & 3.23247100 & -3.01481700\end{array}$

$\begin{array}{lll}5.21104400 & 0.14608100 & -3.63075600\end{array}$

$\begin{array}{lll}5.09549200 & -1.69083000 & -3.45876400\end{array}$

$\begin{array}{lll}6.01828700 & 2.14332900 & -3.64543600\end{array}$

$-3.04523800-0.88462900-4.44067600$

$\begin{array}{lll}1.54331300 & 2.08633100 & -2.45933000\end{array}$

$\begin{array}{llll}1.32724700 & 3.37654100 & -2.80908700\end{array}$

$\begin{array}{llll}0.71861700 & 4.29182200 & -1.93610600\end{array}$

$\begin{array}{llll}0.21059200 & 5.13741200 & -2.39325300\end{array}$

$\begin{array}{lll}1.57894500 & 3.87960100 & -4.17769500\end{array}$

$\begin{array}{llll}2.08543800 & 3.08286400 & -4.73256500\end{array}$

$\begin{array}{lll}0.59187700 & 4.01687700 & -4.64877100\end{array}$

$2.37407400 \quad 5.17303300-4.24149200$

$\begin{array}{lll}1.74414700 & 6.02514000 & -3.94386600\end{array}$

$3.20878300 \quad 5.14466000-3.52265200$

$\begin{array}{llll}2.91294700 & 5.40594600 & -5.64630200\end{array}$

$3.60859200 \quad 4.58975200-5.90279800$

$\begin{array}{lll}2.08540700 & 5.34056300 & -6.37149700\end{array}$

$3.61257800 \quad 6.74684600-5.78119600$

$\begin{array}{lll}4.44396100 & 6.83395400 & -5.06657000\end{array}$

$\begin{array}{llll}4.02491100 & 6.89007300 & -6.78847100\end{array}$

$\begin{array}{lll}2.92026900 & 7.57765900 & -5.58352200\end{array}$

$\begin{array}{llll}0.84272800 & 4.16570200 & -0.60268400\end{array}$

$\begin{array}{lll}1.45323300 & 3.02347100 & 0.15346500\end{array}$

$\begin{array}{lll}0.34002700 & 5.09927400 & 0.25726900\end{array}$

$\begin{array}{lll}1.36935000 & 3.54520900 & 1.55502200\end{array}$

$\begin{array}{lll}2.49239800 & 2.83896400 & -0.17327600\end{array}$

$\begin{array}{lll}0.72178700 & 4.70878500 & 1.52876900\end{array}$

$\begin{array}{lll}1.74460900 & 3.04317200 & 2.43956600\end{array}$

$\begin{array}{lll}0.43094000 & 5.39891800 & 2.31234200\end{array}$

$\begin{array}{lll}6.48926200 & -0.51905100 & -4.03191800\end{array}$

$\begin{array}{llll}6.61460700 & -1.48731000 & -3.53058800\end{array}$

$\begin{array}{lll}6.50620100 & -0.71466400 & -5.11407900\end{array}$

$\begin{array}{llll}7.36031700 & 0.10593400 & -3.79958500\end{array}$

\section{TS III}




\begin{tabular}{|c|c|c|c|}
\hline $\mathrm{C}$ & -2.30597600 & -0.49002400 & -3.82633700 \\
\hline $\mathrm{C}$ & 0.00589600 & 0.27985700 & -4.44374800 \\
\hline $\mathrm{C}$ & -1.67531300 & 2.04998200 & -3.21822600 \\
\hline $\mathrm{O}$ & 0.48826500 & 0.17517800 & -5.49317800 \\
\hline $\mathrm{O}$ & 0.14057100 & -2.33643100 & -1.91073100 \\
\hline $\mathrm{O}$ & -3.12346100 & 0.18574600 & -0.67434600 \\
\hline $\mathrm{O}$ & -2.14607700 & 3.08851000 & -3.42411000 \\
\hline $\mathrm{C}$ & 0.55011700 & 1.69474300 & -0.36897400 \\
\hline $\mathrm{C}$ & -0.24038500 & 1.12558800 & 0.73831700 \\
\hline $\mathrm{C}$ & -1.06934500 & 1.92603900 & 1.53580100 \\
\hline $\mathrm{C}$ & -0.14378800 & -0.23994100 & 1.03555800 \\
\hline $\mathrm{C}$ & -1.78951900 & 1.37431400 & 2.59088000 \\
\hline $\mathrm{H}$ & -1.16572800 & 2.99113400 & 1.31671300 \\
\hline $\mathrm{C}$ & -0.86210200 & -0.79365500 & 2.09136000 \\
\hline $\mathrm{H}$ & 0.51573100 & -0.86581800 & 0.43383200 \\
\hline $\mathrm{C}$ & -1.68839700 & 0.01224900 & 2.87218100 \\
\hline $\mathrm{H}$ & -2.43620800 & 2.00982000 & 3.19501800 \\
\hline $\mathrm{H}$ & -0.77243900 & -1.85780600 & 2.30705600 \\
\hline $\mathrm{H}$ & -2.24995600 & -0.41929000 & 3.69981600 \\
\hline $\mathrm{C}$ & 0.55115100 & 1.36024800 & -1.67605800 \\
\hline $\mathrm{C}$ & 2.76112200 & 1.45946800 & -3.03478800 \\
\hline $\mathrm{C}$ & 2.89105100 & 0.06548600 & -2.96256200 \\
\hline $\mathrm{C}$ & 3.82688200 & 2.18696700 & -3.58506600 \\
\hline $\mathrm{C}$ & 4.01435600 & -0.57430300 & -3.46617300 \\
\hline $\mathrm{H}$ & 2.09632800 & -0.51960800 & -2.50646300 \\
\hline $\mathrm{C}$ & 4.94465300 & 1.54154900 & -4.09751800 \\
\hline $\mathrm{H}$ & 3.79864200 & 3.27609000 & -3.58826100 \\
\hline $\mathrm{C}$ & 5.05653700 & 0.14816300 & -4.05747800 \\
\hline $\mathrm{H}$ & 4.08515000 & -1.66114900 & -3.40422600 \\
\hline $\mathrm{H}$ & 5.75727500 & 2.13163900 & -4.52275500 \\
\hline $\mathrm{O}$ & -3.13603000 & -1.01442000 & -4.45572900 \\
\hline $\mathrm{C}$ & 1.61627600 & 2.14269100 & -2.39457700 \\
\hline $\mathrm{N}$ & 1.20063700 & 3.38569600 & -2.90886400 \\
\hline $\mathrm{C}$ & 0.75289900 & 4.34984700 & -2.05760300 \\
\hline $\mathrm{H}$ & 0.41225300 & 5.28535000 & -2.49315700 \\
\hline $\mathrm{C}$ & 1.22446600 & 3.71577400 & -4.35493700 \\
\hline $\mathrm{H}$ & 1.69110200 & 2.86954500 & -4.86865300 \\
\hline $\mathrm{H}$ & 0.17741000 & 3.75415400 & -4.69043100 \\
\hline $\mathrm{C}$ & 1.93891100 & 5.01145200 & -4.70370600 \\
\hline $\mathrm{H}$ & 1.33920700 & 5.88117900 & -4.39356700 \\
\hline $\mathrm{H}$ & 2.89210900 & 5.07824600 & -4.15464100 \\
\hline $\mathrm{C}$ & 2.20142400 & 5.08986400 & -6.20130700 \\
\hline $\mathrm{H}$ & 2.84327800 & 4.24370900 & -6.49706100 \\
\hline $\mathrm{H}$ & 1.25203800 & 4.95897400 & -6.74532400 \\
\hline $\mathrm{C}$ & 2.85291100 & 6.40075800 & -6.60467100 \\
\hline $\mathrm{H}$ & 3.81592300 & 6.53996400 & -6.09211400 \\
\hline $\mathrm{H}$ & 3.04439600 & 6.44080000 & -7.68472600 \\
\hline $\mathrm{H}$ & 2.21513100 & 7.25876100 & -6.34809300 \\
\hline $\mathrm{C}$ & 0.91020100 & 4.17678500 & -0.71074500 \\
\hline $\mathrm{C}$ & 1.37211400 & 2.94852900 & -0.09941200 \\
\hline $\mathrm{O}$ & 0.94882300 & 5.21475200 & 0.17242900 \\
\hline $\mathrm{C}$ & 1.80917800 & 3.39775700 & 1.21714700 \\
\hline $\mathrm{H}$ & 2.16629300 & 2.61412000 & -1.13834200 \\
\hline $\mathrm{C}$ & 1.51776100 & 4.71130300 & 1.30760400 \\
\hline $\mathrm{H}$ & 2.21475400 & 2.77827800 & 2.00894500 \\
\hline $\mathrm{H}$ & 1.63730800 & 5.42984000 & 2.11049300 \\
\hline $\mathrm{C}$ & 6.25056700 & -0.55522800 & -4.62257500 \\
\hline $\mathrm{H}$ & 6.66239200 & -1.28309000 & -3.91039500 \\
\hline $\mathrm{H}$ & 5.98636000 & -1.11572900 & -5.53079900 \\
\hline $\mathrm{H}$ & 7.04688800 & 0.15085800 & -4.88749700 \\
\hline
\end{tabular}

\section{III}

\begin{tabular}{|c|c|c|c|}
\hline \multicolumn{4}{|c|}{$E=-1749.646729 H$} \\
\hline $\mathrm{Cr}$ & -0.41437000 & 0.50681900 & -2.75675000 \\
\hline $\mathrm{C}$ & -1.82049000 & -0.03637800 & -1.61603900 \\
\hline $\mathrm{C}$ & 0.39148300 & -1.15192800 & -2.35748400 \\
\hline $\mathrm{C}$ & -1.52053900 & -0.17546200 & -4.05887000 \\
\hline $\mathrm{C}$ & 0.72126700 & 0.73080000 & -4.25091200 \\
\hline $\mathrm{C}$ & -1.19146500 & 2.22077700 & -2.78411400 \\
\hline $\mathrm{O}$ & 1.26145000 & 0.74396400 & -5.27821700 \\
\hline $\mathrm{O}$ & 0.82273200 & -2.19482500 & -2.09750600 \\
\hline $\mathrm{O}$ & -2.77562900 & -0.46792800 & -1.12233500 \\
\hline $\mathrm{O}$ & -1.69053400 & 3.26481600 & -2.70819900 \\
\hline $\mathrm{C}$ & 0.50117200 & 1.93548500 & -0.06563700 \\
\hline $\mathrm{C}$ & -0.52818400 & 1.19100800 & 0.71433300 \\
\hline $\mathrm{C}$ & -1.71875000 & 1.80515400 & 1.12111400 \\
\hline $\mathrm{C}$ & -0.28204100 & -0.12688900 & 1.11600600 \\
\hline $\mathrm{C}$ & -2.64753300 & 1.11375200 & 1.89330300 \\
\hline $\mathrm{H}$ & -1.92174600 & 2.83463200 & 0.81979000 \\
\hline $\mathrm{C}$ & -1.20683100 & -0.81895100 & 1.89347000 \\
\hline $\mathrm{H}$ & 0.65236600 & -0.60650900 & 0.81996400 \\
\hline $\mathrm{C}$ & -2.39389100 & -0.20117400 & 2.28132100 \\
\hline $\mathrm{H}$ & -3.57531100 & 1.60202600 & 2.18993700 \\
\hline $\mathrm{H}$ & -0.99895000 & -1.84496900 & 2.19523400 \\
\hline $\mathrm{H}$ & -3.12048800 & -0.74268900 & 2.88582100 \\
\hline $\mathrm{C}$ & 0.87872500 & 1.56884400 & -1.34527900 \\
\hline $\mathrm{C}$ & 3.15146200 & 1.49833700 & -2.63562600 \\
\hline $\mathrm{C}$ & 3.29111000 & 0.12833700 & -2.40310000 \\
\hline $\mathrm{C}$ & 4.04734700 & 2.10033700 & -3.52186700 \\
\hline $\mathrm{C}$ & 4.23259400 & -0.62797700 & -3.09085900 \\
\hline $\mathrm{H}$ & 2.66161000 & -0.35196400 & -1.65609300 \\
\hline $\mathrm{C}$ & 4.98711900 & 1.34163600 & -4.21517500 \\
\hline $\mathrm{H}$ & 4.03431400 & 3.17780000 & -3.68093200 \\
\hline $\mathrm{C}$ & 5.08570600 & -0.03916400 & -4.02909600 \\
\hline $\mathrm{H}$ & 4.30982600 & -1.69810800 & -2.89093100 \\
\hline $\mathrm{H}$ & 5.66478000 & 1.83748200 & -4.91168600 \\
\hline $\mathrm{O}$ & -2.21348100 & -0.59122500 & -4.89894800 \\
\hline $\mathrm{C}$ & 2.17800100 & 2.26701900 & -1.77512400 \\
\hline $\mathrm{N}$ & 1.83175300 & 3.62077200 & -2.27397700 \\
\hline $\mathrm{C}$ & 1.64640500 & 4.56600100 & -1.36937500 \\
\hline $\mathrm{H}$ & 1.53409200 & 5.59293900 & -1.71682400 \\
\hline $\mathrm{C}$ & 1.54619700 & 3.91808700 & -3.69569000 \\
\hline $\mathrm{H}$ & 1.84987200 & 3.04100600 & -4.27227300 \\
\hline $\mathrm{H}$ & 0.45952600 & 4.02386200 & -3.81588800 \\
\hline $\mathrm{C}$ & 2.25686100 & 5.15605400 & -4.22197500 \\
\hline $\mathrm{H}$ & 1.80264300 & 6.07168300 & -3.81289200 \\
\hline $\mathrm{H}$ & 3.31004600 & 5.15585400 & -3.89709500 \\
\hline $\mathrm{C}$ & 2.18774500 & 5.19898800 & -5.74275100 \\
\hline $\mathrm{H}$ & 2.66900300 & 4.29430600 & -6.14994800 \\
\hline $\mathrm{H}$ & 1.13428400 & 5.15603200 & -6.06224200 \\
\hline $\mathrm{C}$ & 2.85305800 & 6.44045000 & -6.30985200 \\
\hline $\mathrm{H}$ & 3.91319500 & 6.48728000 & -6.02167000 \\
\hline $\mathrm{H}$ & 2.80648500 & 6.45870700 & -7.40626400 \\
\hline $\mathrm{H}$ & 2.36859300 & 7.35571900 & -5.94060500 \\
\hline $\mathrm{C}$ & 1.52375900 & 4.26928500 & -0.01421200 \\
\hline $\mathrm{C}$ & 1.00168000 & 3.11558800 & 0.60489300 \\
\hline $\mathrm{O}$ & 1.66084300 & 5.28120600 & 0.89996400 \\
\hline $\mathrm{C}$ & 0.84035800 & 3.49256600 & 1.97679000 \\
\hline $\mathrm{H}$ & 2.75624600 & 2.48946400 & -0.86267000 \\
\hline $\mathrm{C}$ & 1.26356700 & 4.77769400 & 2.08640600 \\
\hline
\end{tabular}




$\begin{array}{rrrr}\mathrm{H} & 0.46190100 & 2.87344700 & 2.78181700 \\ \mathrm{H} & 1.34555200 & 5.44970700 & 2.93256100 \\ \mathrm{C} & 6.06925700 & -0.85938100 & -4.80621300 \\ \mathrm{H} & 6.40174300 & -1.73653600 & -4.23635500 \\ \mathrm{H} & 5.62437400 & -1.23203800 & -5.74043100 \\ \mathrm{H} & 6.95541100 & -0.27335300 & -5.08213600\end{array}$

\section{TS IV}

$\begin{array}{lrrr}\text { E }=-1749.611092 \mathrm{H} & & \\ \mathrm{Cr} & -0.65140500 & 0.19251300 & -2.49828600 \\ \mathrm{C} & -1.79297500 & -0.45286400 & -1.13633600 \\ \mathrm{C} & 0.55224300 & -1.19341200 & -2.05490700 \\ \mathrm{C} & -1.79210700 & -0.79167500 & -3.54639500 \\ \mathrm{C} & 0.05358800 & 0.60398400 & -4.19662600 \\ \mathrm{C} & -1.65713200 & 1.78315500 & -2.51841800 \\ \mathrm{O} & 0.30657600 & 0.76664200 & -5.31934500 \\ \mathrm{O} & 1.27923300 & -2.02428100 & -1.70648800 \\ \mathrm{O} & -2.61421400 & -0.94070300 & -0.48043200 \\ \mathrm{O} & -2.25731100 & 2.77339800 & -2.47367200 \\ \mathrm{C} & 0.33418500 & 2.03086800 & -0.12772600 \\ \mathrm{C} & -0.46112900 & 1.25255000 & 0.85286200 \\ \mathrm{C} & -1.66654000 & 1.73388300 & 1.37985700 \\ \mathrm{C} & 0.04030900 & 0.03111600 & 1.32113800 \\ \mathrm{C} & -2.36332100 & 0.99961000 & 2.33382600 \\ \mathrm{H} & -2.07178200 & 2.68234100 & 1.02391500 \\ \mathrm{C} & -0.64538900 & -0.69162200 & 2.29156000 \\ \mathrm{H} & 0.98619900 & -0.34051200 & 0.92616500 \\ \mathrm{C} & -1.85258600 & -0.21222700 & 2.79617900 \\ \mathrm{H} & -3.31004600 & 1.37659600 & 2.71910400 \\ \mathrm{H} & -0.23729600 & -1.63496000 & 2.65248000 \\ \mathrm{H} & -2.39449900 & -0.78069300 & 3.55085700 \\ \mathrm{C} & 0.73837200 & 1.48615800 & -1.34954400 \\ \mathrm{C} & 3.01386300 & 1.65984900 & -2.70114800 \\ \mathrm{C} & 3.02289000 & 0.50943800 & -3.50401400 \\ \mathrm{C} & 4.17163300 & 2.46238300 & -2.71047600 \\ \mathrm{C} & 4.11335900 & 0.20969800 & -4.30749900 \\ \mathrm{H} & 2.17705000 & -0.16773500 & -3.49993100 \\ \mathrm{C} & 5.25494800 & 2.16806000 & -3.52097000 \\ \mathrm{H} & 4.20247100 & 3.34771500 & -2.07268000 \\ \mathrm{C} & 5.24045000 & 1.03910400 & -4.34845000 \\ \mathrm{H} & 4.09215200 & -0.69209300 & -4.92071500 \\ \mathrm{H} & 6.12977500 & 2.81964400 & -3.51590700 \\ \mathrm{O} & -2.50753100 & -1.41295200 & -4.22646900 \\ \mathrm{C} & 1.94945300 & 2.10828600 & -1.80570400 \\ \mathrm{~N} & 1.18679800 & 3.84651200 & -2.80455800 \\ \mathrm{C} & 0.74699700 & 4.70225200 & -1.95721300 \\ \mathrm{H} & 0.28605000 & 5.63950800 & -2.30481200 \\ \mathrm{C} & 0.87669600 & 4.03436000 & -4.21496300 \\ \mathrm{H} & 0.18349600 & 3.22847300 & -4.49885300 \\ \mathrm{H} & 0.33210900 & 4.98633700 & -4.34813800 \\ \mathrm{C} & 2.10544300 & 3.99763700 & -5.10809200 \\ \mathrm{H} & 2.60649800 & 3.02097800 & -5.01754400 \\ \mathrm{H} & 1.75100900 & 4.06726500 & -6.14901300 \\ \mathrm{C} & 3.09636700 & 5.11820800 & -4.83055700 \\ \mathrm{H} & 2.56372800 & 6.08455500 & -4.82696200 \\ \mathrm{H} & 3.51741800 & 4.99576000 & -3.81916000 \\ \mathrm{C} & 4.22197600 & 5.15191600 & -5.84987000 \\ \mathrm{H} & 3.83303600 & 5.32355300 & -6.86405100 \\ \mathrm{H} & 4.94766600 & 5.94674300 & -5.63234000\end{array}$

$\begin{array}{lrrr}\mathrm{H} & 4.76914800 & 4.19683700 & -5.86718300 \\ \mathrm{C} & 0.77661000 & 4.51040100 & -0.53446000 \\ \mathrm{C} & 0.60376800 & 3.39950200 & 0.28933800 \\ \mathrm{O} & 0.79012400 & 5.64592900 & 0.21249500 \\ \mathrm{C} & 0.53953400 & 3.93409800 & 1.62252000 \\ \mathrm{H} & 2.38802600 & 2.80770500 & -1.08859200 \\ \mathrm{C} & 0.67070700 & 5.27669300 & 1.51157500 \\ \mathrm{H} & 0.41716900 & 3.37455700 & 2.54253500 \\ \mathrm{H} & 0.70559600 & 6.08180000 & 2.23496100 \\ \mathrm{C} & 6.38351300 & 0.73232400 & -5.26177200 \\ \mathrm{H} & 6.13266200 & 0.99875800 & -6.29932000 \\ \mathrm{H} & 7.28466500 & 1.29341400 & -4.98599500 \\ \mathrm{H} & 6.62686400 & -0.33822100 & -5.25871100\end{array}$

IV

$\begin{array}{lrrr}\text { E }=-1749.628757 \mathrm{H} & & \\ \mathrm{Cr} & -0.88396400 & 1.12719300 & -2.43029200 \\ \mathrm{C} & -2.20963700 & 0.25934000 & -1.40118800 \\ \mathrm{C} & 0.17451700 & -0.43799200 & -2.24712500 \\ \mathrm{C} & -1.77971900 & 0.35612400 & -3.85204700 \\ \mathrm{C} & 0.05989200 & 1.95643200 & -3.87361100 \\ \mathrm{C} & -2.02234100 & 2.65246300 & -2.42870000 \\ \mathrm{O} & 0.43508800 & 2.38809400 & -4.87308300 \\ \mathrm{O} & 0.76747900 & -1.41566500 & -2.09806600 \\ \mathrm{O} & -3.08771600 & -0.32151500 & -0.92746600 \\ \mathrm{O} & -2.77092700 & 3.52798800 & -2.44298800 \\ \mathrm{C} & -0.06617600 & 2.03659400 & -0.24190500 \\ \mathrm{C} & -0.23155600 & 0.94452100 & 0.77619600 \\ \mathrm{C} & -1.44027600 & 0.71865000 & 1.44963400 \\ \mathrm{C} & 0.86950800 & 0.14650400 & 1.10893000 \\ \mathrm{C} & -1.54644700 & -0.27165900 & 2.41800800 \\ \mathrm{H} & -2.31904000 & 1.31104600 & 1.19433800 \\ \mathrm{C} & 0.76632400 & -0.84354300 & 2.08316100 \\ \mathrm{H} & 1.82133800 & 0.30030400 & 0.60216300 \\ \mathrm{C} & -0.44068900 & -1.05734300 & 2.74180800 \\ \mathrm{H} & -2.50099400 & -0.43484700 & 2.91665500 \\ \mathrm{H} & 1.63897200 & -1.44799000 & 2.32712400 \\ \mathrm{H} & -0.52396300 & -1.83432600 & 3.50033200 \\ \mathrm{C} & 0.86120800 & 1.85636700 & -1.25531000 \\ \mathrm{C} & 3.13154000 & 1.84335100 & -2.48280600 \\ \mathrm{C} & 2.97903200 & 0.96026400 & -3.56085400 \\ \mathrm{C} & 4.30900400 & 2.60675300 & -2.43569100 \\ \mathrm{C} & 3.94242100 & 0.87693700 & -4.55905500 \\ \mathrm{H} & 2.10400900 & 0.31794600 & -3.62924000 \\ \mathrm{C} & 5.26494500 & 2.52912900 & -3.44012300 \\ \mathrm{H} & 4.45859300 & 3.29561000 & -1.60235400 \\ \mathrm{C} & 5.09907000 & 1.66280900 & -4.52460600 \\ \mathrm{H} & 3.79381100 & 0.18140800 & -5.38667500 \\ \mathrm{H} & 6.15792700 & 3.15431200 & -3.38624300 \\ \mathrm{O} & -2.31012300 & -0.13357200 & -4.75713100 \\ \mathrm{C} & 2.17456300 & 2.03186700 & -1.38949600 \\ \mathrm{~N} & 0.50303700 & 4.55654000 & -2.74982100 \\ \mathrm{C} & 0.13261000 & 5.19907200 & -1.71632500 \\ \mathrm{H} & 0.10879300 & 6.30396800 & -1.70124900 \\ \mathrm{C} & 0.90866500 & 5.33982600 & -3.89855000 \\ \mathrm{H} & 0.28356600 & 5.03352200 & -4.75346300 \\ \mathrm{H} & 0.73984500 & 6.42251000 & -3.73735700 \\ \mathrm{C} & 2.36805800 & 5.07637900 & -4.23587100 \\ \mathrm{H} & 2.99814200 & 5.42061200 & -3.39720800\end{array}$




$\begin{array}{lrrr} & & & \\ \mathrm{H} & 2.52082300 & 3.98801800 & -4.30747300 \\ \mathrm{C} & 2.80778700 & 5.74678000 & -5.52798200 \\ \mathrm{H} & 2.15867700 & 5.40390100 & -6.35066100 \\ \mathrm{H} & 2.65560900 & 6.83605100 & -5.45116500 \\ \mathrm{C} & 4.25951100 & 5.45040900 & -5.86611100 \\ \mathrm{H} & 4.42048000 & 4.36923400 & -5.99628400 \\ \mathrm{H} & 4.57203800 & 5.94804600 & -6.79398900 \\ \mathrm{H} & 4.93289500 & 5.78878600 & -5.06435100 \\ \mathrm{C} & -0.31639000 & 4.62747400 & -0.46129700 \\ \mathrm{C} & -0.49300900 & 3.39184300 & 0.16074100 \\ \mathrm{O} & -0.72391700 & 5.61926700 & 0.38671100 \\ \mathrm{C} & -1.02506500 & 3.70542100 & 1.46038000 \\ \mathrm{H} & 2.63933800 & 2.46223800 & -0.49011200 \\ \mathrm{C} & -1.13980100 & 5.05247000 & 1.53304700 \\ \mathrm{H} & -1.25641400 & 3.01728700 & 2.26195600 \\ \mathrm{H} & -1.48218000 & 5.72717700 & 2.30769200 \\ \mathrm{C} & 6.13445100 & 1.55585600 & -5.60175400 \\ \mathrm{H} & 6.78125900 & 0.67997700 & -5.44614700 \\ \mathrm{H} & 5.67451800 & 1.44222500 & -6.59251600 \\ \mathrm{H} & 6.78305800 & 2.44109100 & -5.62582400\end{array}$

\section{TS V}

$\begin{array}{lrrr}\mathrm{E}=-1749.623606 \mathrm{H} & & \\ \mathrm{Cr} & -0.96352500 & 0.85291700 & -2.24926000 \\ \mathrm{C} & -1.99401500 & -0.25899800 & -1.11197700 \\ \mathrm{C} & 0.19048400 & -0.64931700 & -2.36259900 \\ \mathrm{C} & -2.05180300 & 0.21244200 & -3.60134800 \\ \mathrm{C} & -0.19263300 & 1.85697300 & -3.66974900 \\ \mathrm{C} & -2.17429000 & 2.26717400 & -1.87618200 \\ \mathrm{O} & 0.13223900 & 2.43549400 & -4.61375300 \\ \mathrm{O} & 0.85068100 & -1.59484600 & -2.34713700 \\ \mathrm{O} & -2.68044200 & -0.99721600 & -0.55117900 \\ \mathrm{O} & -2.95620600 & 3.08749300 & -1.65697500 \\ \mathrm{C} & -0.01744700 & 1.87237600 & -0.04927400 \\ \mathrm{C} & -0.43364900 & 0.89981700 & 0.99374300 \\ \mathrm{C} & -1.64827900 & 1.02243500 & 1.68236600 \\ \mathrm{C} & 0.42300900 & -0.16019600 & 1.31911000 \\ \mathrm{C} & -1.98903300 & 0.11842500 & 2.68089600 \\ \mathrm{H} & -2.35282100 & 1.80733500 & 1.40447200 \\ \mathrm{C} & 0.08083700 & -1.06381800 & 2.31978800 \\ \mathrm{H} & 1.36946400 & -0.26949900 & 0.78908700 \\ \mathrm{C} & -1.12407200 & -0.92563400 & 3.00635400 \\ \mathrm{H} & -2.94197000 & 0.22148000 & 3.19795000 \\ \mathrm{H} & 0.76045600 & -1.87849800 & 2.56523700 \\ \mathrm{H} & -1.39220500 & -1.63343700 & 3.78919600 \\ \mathrm{C} & 0.82766600 & 1.50511200 & -1.09304400 \\ \mathrm{C} & 3.05375500 & 1.61084300 & -2.41033500 \\ \mathrm{C} & 2.82463200 & 0.93140000 & -3.61704500 \\ \mathrm{C} & 4.26554600 & 2.31151900 & -2.29001700 \\ \mathrm{C} & 3.74696000 & 0.98074100 & -4.65353000 \\ \mathrm{H} & 1.91853500 & 0.35051100 & -3.76323800 \\ \mathrm{C} & 5.18311000 & 2.36569300 & -3.33193200 \\ \mathrm{H} & 4.47744000 & 2.84583000 & -1.36246100 \\ \mathrm{C} & 4.94007900 & 1.70425200 & -4.53825100 \\ \mathrm{H} & 3.53752100 & 0.44137000 & -5.57897700 \\ \mathrm{H} & 6.10673400 & 2.93272000 & -3.20679100 \\ \mathrm{O} & -2.71841000 & -0.17453700 & -4.46577000 \\ \mathrm{C} & 2.13767000 & 1.70736100 & -1.27281600\end{array}$

$\begin{array}{llll}1.80385600 & 4.85494300 & -2.23382100\end{array}$ $\begin{array}{llll}0.61368600 & 4.52758800 & -1.94693600\end{array}$ $\begin{array}{llll}-0.15777100 & 4.36943900 & -2.72082200\end{array}$ $\begin{array}{llll}2.14848400 & 4.98263600 & -3.64095700\end{array}$ $\begin{array}{llll}2.59074900 & 4.01748000 & -3.94775700\end{array}$ $\begin{array}{llll}1.25111800 & 5.13632500 & -4.26869700\end{array}$ $\begin{array}{llll}3.16394900 & 6.08868100 & -3.85645200\end{array}$ $\begin{array}{llll}2.74347300 & 7.04600700 & -3.50572900\end{array}$ $4.04469300 \quad 5.88701300 \quad-3.22365000$ $\begin{array}{llll}3.58972100 & 6.21225100 & -5.31121000\end{array}$ $3.97910400 \quad 5.24094900-5.66117100$ $\begin{array}{llll}2.70687100 & 6.42870500 & -5.93491800\end{array}$ $\begin{array}{llll}4.64095000 & 7.28968600 & -5.51626600\end{array}$ $\begin{array}{llll}5.54755900 & 7.07351900 & -4.93251100\end{array}$ $\begin{array}{llll}4.93723600 & 7.37592700 & -6.57007100\end{array}$ $\begin{array}{llll}4.26909000 & 8.27305500 & -5.19341200\end{array}$ $\begin{array}{llll}0.17911300 & 4.37340000 & -0.54744300\end{array}$ $\begin{array}{lll}-0.19354900 & 3.30026500 & 0.23921500\end{array}$ $\begin{array}{lll}0.04659500 & 5.52624900 & 0.14281000\end{array}$ $\begin{array}{llll}-0.57196000 & 3.87928100 & 1.50605700\end{array}$ $\begin{array}{llll}2.63630600 & 2.10497300 & -0.37704800\end{array}$ $\begin{array}{lll}-0.40283000 & 5.21481600 & 1.38831000\end{array}$ $\begin{array}{lll}-0.88089400 & 3.35610200 & 2.40224700\end{array}$ $\begin{array}{lll}-0.54086900 & 6.04813000 & 2.06486200\end{array}$ $\begin{array}{llll}5.91199600 & 1.76562300 & -5.67612200\end{array}$ $\begin{array}{llll}6.22270400 & 0.76138600 & -5.99627400\end{array}$ $\begin{array}{llll}5.46626600 & 2.25407700 & -6.55459900\end{array}$ $\begin{array}{llll}6.81413000 & 2.32754800 & -5.40441300\end{array}$

\section{V}

$\begin{array}{lrrr}\text { E }=-1749.628995 \mathrm{H} & & \\ \mathrm{Cr} & -1.02627100 & 0.80807500 & -2.17700300 \\ \mathrm{C} & -2.35883500 & 0.12181000 & -1.02481200 \\ \mathrm{C} & -0.17717400 & -0.88215300 & -2.01790700 \\ \mathrm{C} & -2.09815200 & 0.14120900 & -3.52938700 \\ \mathrm{C} & 0.00061700 & 1.45312100 & -3.65765700 \\ \mathrm{C} & -2.00620200 & 2.43497400 & -2.17076100 \\ \mathrm{O} & 0.44631300 & 1.85579400 & -4.64083000 \\ \mathrm{O} & 0.26411100 & -1.93930100 & -1.88722500 \\ \mathrm{O} & -3.24517600 & -0.34747900 & -0.45404600 \\ \mathrm{O} & -2.68271900 & 3.36890500 & -2.17597500 \\ \mathrm{C} & -0.04483500 & 1.76624800 & 0.04822700 \\ \mathrm{C} & -0.31587600 & 0.75746800 & 1.11279500 \\ \mathrm{C} & -1.50423100 & 0.74854000 & 1.85674800 \\ \mathrm{C} & 0.66363000 & -0.20069000 & 1.40953400 \\ \mathrm{C} & -1.70384200 & -0.18160000 & 2.86916500 \\ \mathrm{H} & -2.29843200 & 1.45603000 & 1.62068200 \\ \mathrm{C} & 0.46601900 & -1.12825500 & 2.42721100 \\ \mathrm{H} & 1.59438800 & -0.21316500 & 0.84333400 \\ \mathrm{C} & -0.71726000 & -1.12194700 & 3.16167400 \\ \mathrm{H} & -2.63932900 & -0.17728500 & 3.42650200 \\ \mathrm{H} & 1.24345800 & -1.85904900 & 2.64466700 \\ \mathrm{H} & -0.87328100 & -1.85165500 & 3.95470700 \\ \mathrm{C} & 0.78942300 & 1.43366000 & -1.00971800 \\ \mathrm{C} & 2.87878800 & 1.29575200 & -2.46726800 \\ \mathrm{C} & 2.83919100 & 0.09900500 & -3.19207800 \\ \mathrm{C} & 3.73262200 & 2.30988600 & -2.91855600 \\ \mathrm{C} & 3.59282400 & -0.05802900 & -4.34905800 \\ \mathrm{H} & 2.22771900 & -0.72843900 & -2.83888400\end{array}$




$\begin{array}{lrrr}\mathrm{C} & 4.47187200 & 2.15539300 & -4.08680700 \\ \mathrm{H} & 3.80274200 & 3.24045600 & -2.35167800 \\ \mathrm{C} & 4.41197200 & 0.97077300 & -4.82654100 \\ \mathrm{H} & 3.54734000 & -1.00159300 & -4.89578400 \\ \mathrm{H} & 5.11329300 & 2.96848300 & -4.42986100 \\ \mathrm{O} & -2.75630800 & -0.27572000 & -4.38560200 \\ \mathrm{C} & 2.09495100 & 1.54556400 & -1.24749100 \\ \mathrm{~N} & 0.61476200 & 5.66525900 & -2.28978300 \\ \mathrm{C} & 0.46195800 & 4.50538900 & -1.78143000 \\ \mathrm{H} & 0.72999000 & 3.59188500 & -2.33232900 \\ \mathrm{C} & 1.10843900 & 5.71237800 & -3.65246200 \\ \mathrm{H} & 1.84058000 & 6.53097100 & -3.72468200 \\ \mathrm{H} & 1.62694100 & 4.77541500 & -3.93219300 \\ \mathrm{C} & -0.03233800 & 5.96903500 & -4.62863800 \\ \mathrm{H} & -0.76589400 & 5.14882200 & -4.53899800 \\ \mathrm{H} & -0.56178400 & 6.89111300 & -4.33860900 \\ \mathrm{C} & 0.44976300 & 6.06860700 & -6.06778000 \\ \mathrm{H} & 1.17845500 & 6.89108100 & -6.15239900 \\ \mathrm{H} & 0.99477800 & 5.14721100 & -6.33159900 \\ \mathrm{C} & -0.69171200 & 6.28744600 & -7.04614000 \\ \mathrm{H} & -1.23826900 & 7.21444700 & -6.81934700 \\ \mathrm{H} & -0.33271100 & 6.35823000 & -8.08148800 \\ \mathrm{H} & -1.41554600 & 5.46070800 & -7.00189500 \\ \mathrm{C} & -0.07004300 & 4.30292200 & -0.44759000 \\ \mathrm{C} & -0.35143600 & 3.17828600 & 0.32577600 \\ \mathrm{O} & -0.40995300 & 5.42178700 & 0.23343900 \\ \mathrm{C} & -0.87877700 & 3.69887300 & 1.55886800 \\ \mathrm{H} & 2.67564600 & 1.97515000 & -0.41762900 \\ \mathrm{C} & -0.88584800 & 5.04743800 & 1.44000100 \\ \mathrm{H} & -1.17272500 & 3.14779900 & 2.44190100 \\ \mathrm{H} & -1.17877400 & 5.84956000 & 2.10559200 \\ \mathrm{C} & 5.21177300 & 0.78993900 & -6.08027100 \\ \mathrm{H} & 5.94872100 & -0.01852600 & -5.97285500 \\ \mathrm{H} & 4.56956000 & 0.52241800 & -6.93077300 \\ \mathrm{H} & 5.75727000 & 1.70394300 & -6.34565500\end{array}$

\section{TS VI}

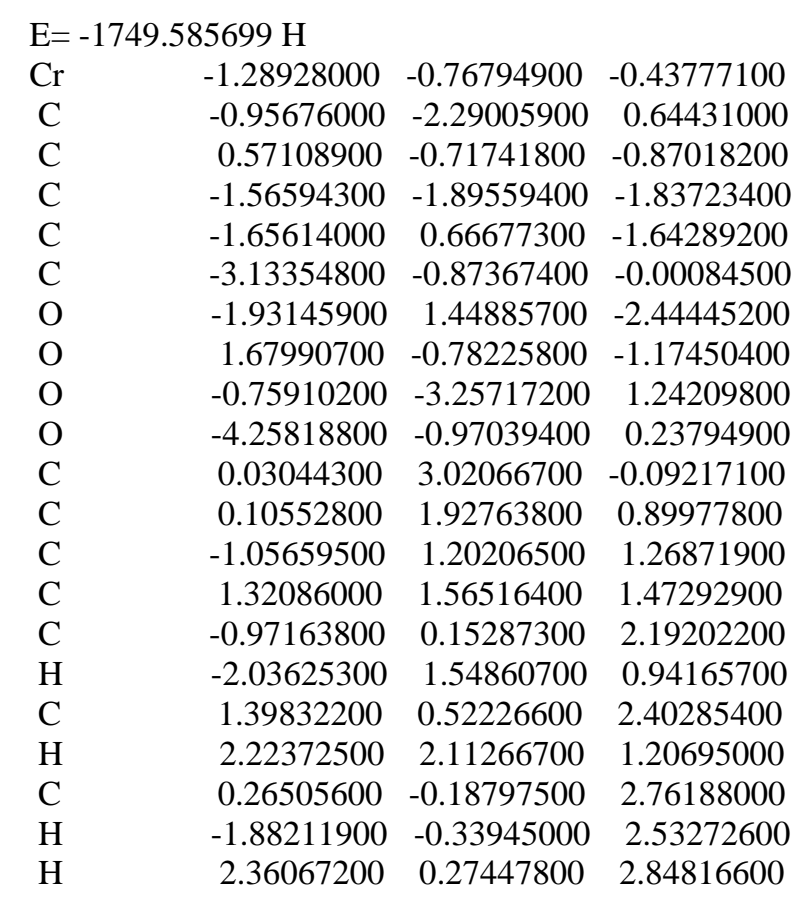

$\begin{array}{lll}0.32458700 & -0.99664900 & 3.48854300\end{array}$

$\begin{array}{llll}0.89681700 & 3.05235700 & -1.17939400\end{array}$

$\begin{array}{llll}2.72640700 & 2.57275800 & -2.80324500\end{array}$

$\begin{array}{lll}1.94463200 & 1.95224500 & -3.79281000\end{array}$

$\begin{array}{lll}4.06542100 & 2.85875200 & -3.10868900\end{array}$

$\begin{array}{llll}2.47826000 & 1.65918600 & -5.04040100\end{array}$

$\begin{array}{llll}0.91596500 & 1.66911500 & -3.56315700\end{array}$

$\begin{array}{lll}4.59373400 & 2.56262400 & -4.35968900\end{array}$

$\begin{array}{lll}4.69432800 & 3.32803500 & -2.35046800\end{array}$

$\begin{array}{lll}3.81065900 & 1.96074700 & -5.35004500\end{array}$

$\begin{array}{lll}1.85411900 & 1.17097900 & -5.79144400\end{array}$

$\begin{array}{llll}5.63691500 & 2.79870600 & -4.57504700\end{array}$

$\begin{array}{lll}-1.74060900 & -2.62050200 & -2.72950000\end{array}$

$\begin{array}{lll}2.19627100 & 2.89849300 & -1.48680600\end{array}$

$-0.41112500 \quad 5.36497500 \quad-3.47132600$

$\begin{array}{lll}-0.63497900 & 4.62484500 & -2.49006700\end{array}$

$\begin{array}{llll}0.14846800 & 3.46815300 & -2.20914400\end{array}$

$\begin{array}{llll}0.12409400 & 4.85951700 & -4.72750500\end{array}$

$\begin{array}{lll}0.09020600 & 3.75692600 & -4.75200300\end{array}$

$\begin{array}{lll}-0.52845800 & 5.23866700 & -5.52868700\end{array}$

$\begin{array}{lll}1.54748500 & 5.34448100 & -4.95614800\end{array}$

$2.19478000 \quad 4.93301900-4.16233300$

$\begin{array}{lll}1.90176900 & 4.90274300 & -5.90262700\end{array}$

$\begin{array}{lll}1.70244600 & 6.85637200 & -5.01014200\end{array}$

$\begin{array}{llll}1.03195600 & 7.26599600 & -5.78375700\end{array}$

$\begin{array}{lll}1.37229500 & 7.29437200 & -4.05590700\end{array}$

$\begin{array}{lll}3.13801900 & 7.26401400 & -5.29702700\end{array}$

$\begin{array}{llll}3.47627300 & 6.87654800 & -6.26954300\end{array}$

$\begin{array}{llll}3.25911800 & 8.35531500 & -5.31435700\end{array}$

$\begin{array}{llll}3.82215700 & 6.86666800 & -4.53210800\end{array}$

$\begin{array}{llll}-1.32838200 & 4.69223000 & -1.28051700\end{array}$

$\begin{array}{llll}-1.07332700 & 3.90926400 & -0.14436600\end{array}$

$\begin{array}{llll}-2.53979700 & 5.31339400 & -1.16287500\end{array}$

$\begin{array}{lll}-2.18859900 & 4.14909300 & 0.73130100\end{array}$

$\begin{array}{lll}2.94215300 & 3.07400900 & -0.70468700\end{array}$

$\begin{array}{lll}-3.02369400 & 4.97884900 & 0.06204900\end{array}$

$\begin{array}{lll}-2.34110900 & 3.76368800 & 1.73280100\end{array}$

$\begin{array}{lll}-3.97741500 & 5.42213600 & 0.32214200\end{array}$

$\begin{array}{lll}4.37557600 & 1.63194000 & -6.69816200\end{array}$

$\begin{array}{lll}3.82963900 & 2.14891000 & -7.50025900\end{array}$

$\begin{array}{llll}5.43114700 & 1.92157300 & -6.77128600\end{array}$

$\begin{array}{llll}4.30763400 & 0.55583700 & -6.91131100\end{array}$

VI

$\begin{array}{lrrr}\mathrm{E}= & -1749.632199 \mathrm{H} & & \\ \mathrm{C} & -0.62281800 & 1.26804300 & 1.76714600 \\ \mathrm{C} & -0.25638400 & -0.08518100 & 1.74235200 \\ \mathrm{C} & -0.58744100 & 1.94277600 & 2.99549700 \\ \mathrm{C} & 0.13515800 & -0.73938900 & 2.90730100 \\ \mathrm{H} & -0.30230000 & -0.63642900 & 0.80256000 \\ \mathrm{C} & -0.20284800 & 1.28903900 & 4.16223500 \\ \mathrm{H} & -0.86075400 & 2.99872300 & 3.03335200 \\ \mathrm{C} & 0.16424300 & -0.05436000 & 4.12132300 \\ \mathrm{H} & 0.41023100 & -1.79283500 & 2.86844400 \\ \mathrm{H} & -0.18021800 & 1.83478000 & 5.10489800 \\ \mathrm{H} & 0.46929800 & -0.56798400 & 5.03215400 \\ \mathrm{C} & -0.22057300 & 2.01252900 & -0.63065900 \\ \mathrm{C} & 1.44387400 & 0.96146200 & -2.20506800 \\ \mathrm{C} & 2.44999700 & -0.00872400 & -2.30398600\end{array}$




$\begin{array}{lrrr}\mathrm{C} & 0.91253400 & 1.46661000 & -3.40302600 \\ \mathrm{C} & 2.91547300 & -0.44730400 & -3.53948900 \\ \mathrm{H} & 2.87619400 & -0.42309600 & -1.38828500 \\ \mathrm{C} & 1.38300500 & 1.02937500 & -4.63333600 \\ \mathrm{H} & 0.11982500 & 2.21680700 & -3.38610300 \\ \mathrm{C} & 2.39840600 & 0.06887600 & -4.72938200 \\ \mathrm{H} & 3.69892300 & -1.20563800 & -3.58052700 \\ \mathrm{H} & 0.95217600 & 1.44288800 & -5.54715100 \\ \mathrm{C} & 0.98550200 & 1.36356400 & -0.86295400 \\ \mathrm{~N} & -2.09894000 & 4.38403100 & -2.71763900 \\ \mathrm{C} & -2.43330100 & 3.99673200 & -1.62440200 \\ \mathrm{H} & -0.69204800 & 2.45226400 & -1.51184900 \\ \mathrm{C} & -1.52987400 & 5.65963800 & -3.16122000 \\ \mathrm{H} & -2.29537600 & 6.42976100 & -2.98721600 \\ \mathrm{H} & -0.66694400 & 5.90495900 & -2.52327500 \\ \mathrm{C} & -1.15084200 & 5.59403100 & -4.62460700 \\ \mathrm{H} & -0.40716600 & 4.79422900 & -4.77352600 \\ \mathrm{H} & -2.04013400 & 5.30930700 & -5.20949400 \\ \mathrm{C} & -0.60032200 & 6.91824300 & -5.12976100 \\ \mathrm{H} & -1.35523600 & 7.70720400 & -4.97864100 \\ \mathrm{H} & 0.27299300 & 7.21070000 & -4.52425900 \\ \mathrm{C} & -0.20955400 & 6.85284600 & -6.59624300 \\ \mathrm{H} & -1.07188200 & 6.58633900 & -7.22456900 \\ \mathrm{H} & 0.17968500 & 7.81372900 & -6.95729400 \\ \mathrm{H} & 0.56871100 & 6.09438100 & -6.76409500 \\ \mathrm{C} & -2.89764300 & 3.42802500 & -0.50716600 \\ \mathrm{C} & -2.29661600 & 2.58562200 & 0.51075000 \\ \mathrm{O} & -4.22130700 & 3.72181500 & -0.16084700 \\ \mathrm{C} & -3.35003500 & 2.46196000 & 1.49453000 \\ \mathrm{H} & 1.40651000 & 0.76417900 & -0.05284700 \\ \mathrm{C} & -4.42830300 & 3.12667300 & 1.03529000 \\ \mathrm{H} & -3.30059900 & 1.89504000 & 2.41655000 \\ \mathrm{H} & -5.42287300 & 3.25461800 & 1.44798000 \\ \mathrm{C} & -1.05180200 & 1.97630600 & 0.53678400 \\ \mathrm{Cr} & 1.91519400 & 3.56171200 & -0.28733100 \\ \mathrm{C} & 2.32274800 & 2.86043000 & 1.43933300 \\ \mathrm{O} & 2.70344500 & 2.48150300 & 2.45955300 \\ \mathrm{C} & 3.50242000 & 2.71804900 & -0.88757200 \\ \mathrm{O} & 4.52073600 & 2.29560100 & -1.22749100 \\ \mathrm{C} & 2.95860100 & 5.01153700 & 0.09939000 \\ \mathrm{O} & 3.61660400 & 5.93711200 & 0.34943300 \\ \mathrm{C} & 0.47040100 & 4.60297800 & 0.37662600 \\ \mathrm{O} & -0.32446800 & 5.32004700 & 0.80947400 \\ \mathrm{C} & 1.68008600 & 4.24349700 & -2.03953300 \\ \mathrm{O} & 1.65397600 & 4.72200100 & -3.09097700 \\ \mathrm{C} & 2.92226500 & -0.36841100 & -6.06288300 \\ \mathrm{H} & 2.11446400 & -0.48476400 & -6.79765700 \\ \mathrm{H} & 3.62647100 & 0.36861100 & -6.47641300 \\ \mathrm{H} & 3.45700900 & -1.32387300 & -5.99025000 \\ & & & \end{array}$

\section{VII}

$\begin{array}{lrrc}\text { E }=-1749.615512 \mathrm{H} & & \\ \mathrm{C} & -0.15437200 & 1.02594300 & 0.83706400 \\ \mathrm{C} & -1.09695100 & 0.29104400 & 1.57521000 \\ \mathrm{C} & 1.17745700 & 0.57738400 & 0.85568100 \\ \mathrm{C} & -0.71578600 & -0.81470200 & 2.32910200 \\ \mathrm{H} & -2.14991400 & 0.56645100 & 1.53523200 \\ \mathrm{C} & 1.55851600 & -0.53264300 & 1.60188800\end{array}$

$\begin{array}{lll}1.93133100 & 1.11510400 & 0.27970900\end{array}$

$\begin{array}{lll}0.61445000 & -1.23002100 & 2.35278800\end{array}$

$\begin{array}{lll}-1.47067100 & -1.36675500 & 2.88825700\end{array}$

$\begin{array}{lll}2.60105300 & -0.84884800 & 1.60156100\end{array}$

$\begin{array}{lll}0.90898600 & -2.09725100 & 2.94215400\end{array}$

$\begin{array}{llll}0.12936400 & 2.21003000 & -1.31913200\end{array}$

$\begin{array}{lll}1.13729900 & 3.32529100 & -3.32892400\end{array}$

$\begin{array}{lll}1.74922100 & 4.50829900 & -3.76947200\end{array}$

$\begin{array}{lll}1.12860400 & 2.22686900 & -4.20690200\end{array}$

$\begin{array}{lll}2.33781800 & 4.59135000 & -5.02706000\end{array}$

$\begin{array}{lll}1.76087900 & 5.37875300 & -3.10921900\end{array}$

$\begin{array}{lll}1.72070300 & 2.31453800 & -5.45792300\end{array}$

$\begin{array}{llll}0.63124100 & 1.30159100 & -3.91555400\end{array}$

$\begin{array}{llll}2.33762000 & 3.49586100 & -5.89390400\end{array}$

$\begin{array}{lll}2.80037500 & 5.52681000 & -5.34708900\end{array}$

$\begin{array}{llll}1.69339300 & 1.45201600 & -6.12610100\end{array}$

$\begin{array}{llll}0.51658400 & 3.29933900 & -2.01214700\end{array}$

$\begin{array}{llll}-2.35033500 & 4.60555200 & -2.96408100\end{array}$

$\begin{array}{llll}-2.24290900 & 4.33848700 & -1.78778900\end{array}$

$\begin{array}{llll}0.35713500 & 1.22346900 & -1.73632100\end{array}$

$\begin{array}{llll}-1.76697000 & 5.73537200 & -3.69801900\end{array}$

$\begin{array}{llll}-2.52875100 & 6.52884100 & -3.69241200\end{array}$

$\begin{array}{lll}-0.88331800 & 6.11757300 & -3.16264100\end{array}$

$\begin{array}{llll}-1.42811800 & 5.33421700 & -5.11872200\end{array}$

$\begin{array}{llll}-0.75860100 & 4.45873700 & -5.09658200\end{array}$

$\begin{array}{llll}-2.34850800 & 5.01538600 & -5.63336600\end{array}$

$\begin{array}{lll}-0.75971500 & 6.46707400 & -5.88237600\end{array}$

$\begin{array}{lll}-1.42514700 & 7.34573700 & -5.89916700\end{array}$

$\begin{array}{llll}0.14810900 & 6.78002300 & -5.33995100\end{array}$

$\begin{array}{llll}-0.39298800 & 6.06642600 & -7.30110900\end{array}$

$\begin{array}{lll}-1.28530500 & 5.78521500 & -7.87913100\end{array}$

$\begin{array}{llll}0.10750600 & 6.88303900 & -7.83829800\end{array}$

$\begin{array}{llll}0.28682900 & 5.19995700 & -7.30329800\end{array}$

$\begin{array}{llll}-2.26268500 & 4.02105800 & -0.49176300\end{array}$

$\begin{array}{lll}-1.54524600 & 3.05342300 & 0.33816600\end{array}$

$\begin{array}{lll}-3.26650600 & 4.64443600 & 0.26793300\end{array}$

$\begin{array}{llll}-2.24550600 & 3.14798700 & 1.61217900\end{array}$

$\begin{array}{llll}0.40379200 & 4.28376600 & -1.54592000\end{array}$

$\begin{array}{lll}-3.21346000 & 4.07316500 & 1.49422700\end{array}$

$\begin{array}{lll}-2.00497600 & 2.61131800 & 2.52275400\end{array}$

$\begin{array}{lll}-3.93084400 & 4.45523300 & 2.21244100\end{array}$

$\begin{array}{lll}-0.52331900 & 2.18322500 & -0.01270300\end{array}$

$\begin{array}{lll}0.92041200 & 4.61246700 & 1.70645200\end{array}$

$\begin{array}{llll}1.10857200 & 3.23872400 & 3.02697000\end{array}$

$\begin{array}{lll}1.23706200 & 2.47597000 & 3.87929800\end{array}$

$\begin{array}{lll}2.34884400 & 3.90308400 & 0.65423100\end{array}$

$\begin{array}{lll}3.27309500 & 3.57329100 & 0.05043400\end{array}$

$\begin{array}{lll}2.14651000 & 5.62396900 & 2.57901000\end{array}$

$\begin{array}{lll}2.93671400 & 6.26866700 & 3.13883600\end{array}$

$\begin{array}{lll}-0.42255800 & 5.43799500 & 2.77007000\end{array}$

$\begin{array}{lll}-1.15682300 & 6.00565500 & 3.45472500\end{array}$

$\begin{array}{lll}0.68742800 & 5.99621000 & 0.43438600\end{array}$

$\begin{array}{llll}0.57930900 & 6.87879300 & -0.30184700\end{array}$

$\begin{array}{llll}2.96592300 & 3.57270800 & -7.25169500\end{array}$

$\begin{array}{llll}3.89375400 & 2.98383700 & -7.29687400\end{array}$

$\begin{array}{llll}2.29837600 & 3.17140400 & -8.02685800\end{array}$

$3.21720400 \quad 4.60644300 \quad-7.52116500$

\section{TS VIII}




\begin{tabular}{|c|c|c|c|}
\hline \multicolumn{4}{|c|}{$04035 \mathrm{H}$} \\
\hline C & 0.08820100 & 0.99917100 & 1.05478700 \\
\hline $\mathrm{C}$ & -0.90893700 & 0.34258300 & 1.79326500 \\
\hline $\mathrm{C}$ & 1.42276200 & 0.63091100 & 1.28801200 \\
\hline $\mathrm{C}$ & -0.57987500 & -0.61490200 & 2.74807100 \\
\hline $\mathrm{H}$ & -1.95878600 & 0.55504000 & 1.59614800 \\
\hline C & 1.75376000 & -0.32646700 & 2.24154700 \\
\hline $\mathrm{H}$ & 2.21499100 & 1.11260300 & 0.71501800 \\
\hline C & 0.75255300 & -0.94826400 & 2.98443100 \\
\hline $\mathrm{H}$ & -1.37428500 & -1.11543000 & 3.30054400 \\
\hline $\mathrm{H}$ & 2.79958300 & -0.58244300 & 2.40736300 \\
\hline $\mathrm{H}$ & 1.00733900 & -1.69491400 & 3.73511000 \\
\hline C & 0.46089200 & 1.83073400 & -1.24087400 \\
\hline C & 0.98715300 & 2.53364000 & -3.60103800 \\
\hline C & 1.40282900 & 3.61509200 & -4.38957100 \\
\hline C & 0.99394100 & 1.25509100 & -4.18722100 \\
\hline C & 1.82276300 & 3.43196800 & -5.70351700 \\
\hline $\mathrm{H}$ & 1.40495700 & 4.61641200 & -3.95537100 \\
\hline C & 1.42323800 & 1.07688700 & -5.49319800 \\
\hline $\mathrm{H}$ & 0.64366800 & 0.39380500 & -3.61792500 \\
\hline C & 1.84244500 & 2.16030600 & -6.27918500 \\
\hline $\mathrm{H}$ & 2.13943700 & 4.29327200 & -6.29392900 \\
\hline $\mathrm{H}$ & 1.42186900 & 0.07567200 & -5.92781300 \\
\hline C & 0.52321500 & 2.77344200 & -2.24400400 \\
\hline $\mathrm{N}$ & -1.85235400 & 4.23854600 & -3.42168700 \\
\hline C & -1.66066600 & 3.73436100 & -2.32178300 \\
\hline $\mathrm{H}$ & 0.90693000 & 0.84751400 & -1.42046600 \\
\hline C & -2.12896300 & 5.66369000 & -3.65018500 \\
\hline $\mathrm{H}$ & -3.18399300 & 5.72068100 & -3.95893000 \\
\hline $\mathrm{H}$ & -2.02095300 & 6.22854800 & -2.70836400 \\
\hline C & -1.24747100 & 6.24294000 & -4.73814800 \\
\hline $\mathrm{H}$ & -0.19289200 & 6.20260400 & -4.41982900 \\
\hline $\mathrm{H}$ & -1.32474700 & 5.61418600 & -5.64006300 \\
\hline C & -1.62658500 & 7.67988600 & -5.06473300 \\
\hline $\mathrm{H}$ & -2.67521300 & 7.71116700 & -5.40261000 \\
\hline $\mathrm{H}$ & -1.58267700 & 8.28750200 & -4.14604600 \\
\hline C & -0.72315000 & 8.28494600 & -6.12553600 \\
\hline $\mathrm{H}$ & -0.76838400 & 7.70756400 & -7.06039800 \\
\hline $\mathrm{H}$ & -1.00465400 & 9.32001400 & -6.36003900 \\
\hline $\mathrm{H}$ & 0.32534500 & 8.29293900 & -5.79396800 \\
\hline C & -1.91430000 & 3.61232400 & -0.98455800 \\
\hline C & -1.30038800 & 2.88437600 & 0.08846200 \\
\hline $\mathrm{O}$ & -3.16447700 & 4.06876600 & -0.56953600 \\
\hline C & -2.20865700 & 3.06953600 & 1.20102900 \\
\hline $\mathrm{H}$ & 0.49589000 & 3.82653700 & -1.95030300 \\
\hline C & -3.28528600 & 3.73510600 & 0.73565400 \\
\hline $\mathrm{H}$ & -2.07370900 & 2.72424700 & 2.21947500 \\
\hline $\mathrm{H}$ & -4.20472100 & 4.05047500 & 1.21581300 \\
\hline C & -0.21188700 & 1.98953500 & -0.00698500 \\
\hline $\mathrm{Cr}$ & 0.82375400 & 4.80585300 & 1.39178400 \\
\hline C & 0.84499400 & 3.76327900 & 2.99575400 \\
\hline $\mathrm{O}$ & 0.86600900 & 3.20391500 & 4.00167400 \\
\hline C & 2.41076100 & 3.97352600 & 0.73758900 \\
\hline $\mathrm{O}$ & 3.41514900 & 3.56529600 & 0.34698200 \\
\hline C & 1.85704100 & 6.05918600 & 2.19827300 \\
\hline $\mathrm{O}$ & 2.52022500 & 6.86113800 & 2.71759900 \\
\hline C & -0.70892900 & 5.74902000 & 2.02054000 \\
\hline $\mathrm{O}$ & -1.57724900 & 6.39312000 & 2.42014400 \\
\hline C & 0.77341500 & 5.87630500 & -0.17542100 \\
\hline $\mathrm{O}$ & 0.77044300 & 6.57852100 & -1.09119700 \\
\hline
\end{tabular}

$\begin{array}{llll}\mathrm{C} & 2.27926100 & 1.94984000 & -7.69654600 \\ \mathrm{H} & 2.66176600 & 2.87731600 & -8.14045300 \\ \mathrm{H} & 3.07009600 & 1.19016900 & -7.76623200 \\ \mathrm{H} & 1.44703200 & 1.59917200 & -8.32370900\end{array}$

\section{VIII}

\begin{tabular}{|c|c|c|c|}
\hline \multicolumn{4}{|c|}{$E=-1749.6$} \\
\hline C & 0.22397200 & 1.27661100 & 0.92219900 \\
\hline $\mathrm{C}$ & -0.85784100 & 0.38474700 & 0.94206200 \\
\hline $\mathrm{C}$ & 1.38046500 & 0.90866300 & 1.62585700 \\
\hline $\mathrm{C}$ & -0.79278000 & -0.81175400 & 1.65396700 \\
\hline $\mathrm{H}$ & -1.75535900 & 0.59646200 & 0.36578500 \\
\hline $\mathrm{C}$ & 1.44429000 & -0.27815600 & 2.34177500 \\
\hline $\mathrm{H}$ & 2.25296600 & 1.55593300 & 1.62288800 \\
\hline $\mathrm{C}$ & 0.35218300 & -1.14538200 & 2.36694100 \\
\hline $\mathrm{H}$ & -1.64930700 & -1.48424000 & 1.64329400 \\
\hline $\mathrm{H}$ & 2.35422600 & -0.52570500 & 2.88630000 \\
\hline $\mathrm{H}$ & 0.39949100 & -2.07470700 & 2.93225000 \\
\hline $\mathrm{C}$ & 1.27302100 & 2.95761700 & -0.61854600 \\
\hline $\mathrm{C}$ & 1.44139200 & 2.45252400 & -3.03417600 \\
\hline $\mathrm{C}$ & 2.22818400 & 2.74705700 & -4.15056400 \\
\hline $\mathrm{C}$ & 0.88335100 & 1.17540400 & -2.93391200 \\
\hline $\mathrm{C}$ & 2.44576800 & 1.79379000 & -5.14031300 \\
\hline $\mathrm{H}$ & 2.66777500 & 3.74043600 & -4.25006100 \\
\hline $\mathrm{C}$ & 1.10444300 & 0.22430900 & -3.92667200 \\
\hline $\mathrm{H}$ & 0.26111800 & 0.91028100 & -2.07701800 \\
\hline $\mathrm{C}$ & 1.88921000 & 0.51479000 & -5.04609600 \\
\hline $\mathrm{H}$ & 3.06076000 & 2.04784800 & -6.00515300 \\
\hline $\mathrm{H}$ & 0.65594300 & -0.76554600 & -3.82925900 \\
\hline $\mathrm{C}$ & 1.17979200 & 3.55243000 & -2.00522600 \\
\hline $\mathrm{N}$ & -0.10882700 & 5.16090400 & -3.23530000 \\
\hline $\mathrm{C}$ & -0.13835200 & 4.24239600 & -2.34592200 \\
\hline $\mathrm{H}$ & 2.22883400 & 2.46777700 & -0.42406400 \\
\hline $\mathrm{C}$ & -1.31387600 & 5.83885900 & -3.68206000 \\
\hline $\mathrm{H}$ & -2.02747500 & 5.11307400 & -4.10880900 \\
\hline $\mathrm{H}$ & -1.83144500 & 6.30830400 & -2.82736300 \\
\hline $\mathrm{C}$ & -0.97860300 & 6.89797400 & -4.71523100 \\
\hline $\mathrm{H}$ & -0.24441900 & 7.59894900 & -4.28410200 \\
\hline $\mathrm{H}$ & -0.48029400 & 6.41933400 & -5.57488400 \\
\hline $\mathrm{C}$ & -2.20640100 & 7.66167100 & -5.18543900 \\
\hline $\mathrm{H}$ & -2.94141800 & 6.95229000 & -5.60100000 \\
\hline $\mathrm{H}$ & -2.69668300 & 8.13305900 & -4.31733500 \\
\hline $\mathrm{C}$ & -1.87274700 & 8.72127500 & -6.22204800 \\
\hline $\mathrm{H}$ & -1.41786700 & 8.27213500 & -7.11699300 \\
\hline $\mathrm{H}$ & -2.76528900 & 9.27349300 & -6.54535200 \\
\hline $\mathrm{H}$ & -1.15590500 & 9.45361400 & -5.82241600 \\
\hline $\mathrm{C}$ & -1.24942100 & 3.74294100 & -1.56200000 \\
\hline $\mathrm{C}$ & -1.14311600 & 2.98436300 & -0.41643700 \\
\hline $\mathrm{O}$ & -2.53272200 & 4.15742700 & -1.72929200 \\
\hline $\mathrm{C}$ & -2.45073900 & 2.95565800 & 0.15732200 \\
\hline $\mathrm{H}$ & 1.96809100 & 4.29938300 & -2.15879400 \\
\hline $\mathrm{C}$ & -3.24254100 & 3.67284800 & -0.68471400 \\
\hline $\mathrm{H}$ & -2.76883600 & 2.48856200 & 1.08246300 \\
\hline $\mathrm{H}$ & -4.29819500 & 3.91427600 & -0.67757400 \\
\hline $\mathrm{C}$ & 0.16060700 & 2.49298200 & 0.06151900 \\
\hline $\mathrm{Cr}$ & 1.16286100 & 4.63471600 & 1.24722600 \\
\hline $\mathrm{C}$ & 2.76645200 & 3.89153500 & 1.94208300 \\
\hline $\mathrm{O}$ & 3.77176600 & 3.56510400 & 2.40620800 \\
\hline $\mathrm{C}$ & 2.21775700 & 5.59520700 & 0.00086700 \\
\hline
\end{tabular}




$\begin{array}{lrrr}\mathrm{O} & 2.85643500 & 6.23478900 & -0.71570700 \\ \mathrm{C} & 1.43275600 & 6.00079100 & 2.43865200 \\ \mathrm{O} & 1.60125400 & 6.85419500 & 3.20640600 \\ \mathrm{C} & 0.08115100 & 3.79618800 & 2.57549900 \\ \mathrm{O} & -0.57051600 & 3.36863800 & 3.42437100 \\ \mathrm{C} & -0.35826700 & 5.60176900 & 0.63869300 \\ \mathrm{O} & -1.24752200 & 6.27640900 & 0.34913100 \\ \mathrm{C} & 2.14805500 & -0.52069600 & -6.09834200 \\ \mathrm{H} & 2.27682000 & -0.06572200 & -7.08899100 \\ \mathrm{H} & 3.06527800 & -1.08803200 & -5.88231700 \\ \mathrm{H} & 1.32713400 & -1.24656300 & -6.15966900\end{array}$

\section{$5 b-C r$}

$E=-1749.713575 \mathrm{H}$

$\begin{array}{lrrr}\mathrm{C} & 0.12574200 & 1.11242800 & 0.52147600 \\ \mathrm{C} & -1.00377900 & 0.34500500 & 0.84694600 \\ \mathrm{C} & 1.37384300 & 0.65645400 & 0.97526400 \\ \mathrm{C} & -0.89237800 & -0.81471800 & 1.60870400 \\ \mathrm{H} & -1.98245400 & 0.63619300 & 0.46961000 \\ \mathrm{C} & 1.48733500 & -0.50588200 & 1.72890100 \\ \mathrm{H} & 2.27723800 & 1.22067500 & 0.73969100 \\ \mathrm{C} & 0.35307800 & -1.24652200 & 2.05767000 \\ \mathrm{H} & -1.78748200 & -1.39049100 & 1.84174400\end{array}$

$\begin{array}{llll}\mathrm{H} & 2.46967600 & -0.82918600 & 2.07194300\end{array}$

$\mathrm{H} \quad 0.44047000 \quad-2.15352700 \quad 2.65394200$

C $\quad 1.04094100 \quad 2.68096300 \quad-1.20440200$

C $\quad 2.14241800 \quad 3.96686900 \quad-3.01039600$

$\begin{array}{llll}C & 2.70698500 & 5.24377200 & -3.12467900\end{array}$

C $\quad 2.77840900 \quad 2.90951500 \quad-3.67750600$

C $\quad 3.86279800 \quad 5.45224300 \quad-3.87341200$

$\mathrm{H} \quad 2.24672500 \quad 6.08628500 \quad-2.60657100$

C $\quad 3.93062700 \quad 3.12268600 \quad-4.42383800$

$\mathrm{H} \quad 2.35446000 \quad 1.90655700 \quad-3.61418100$

C $\quad 4.49672000 \quad 4.39797700 \quad-4.53428600$

$\mathrm{H} \quad 4.28576600 \quad 6.45576000 \quad-3.93886100$

$\mathrm{H} \quad 4.40411100 \quad 2.28322900 \quad-4.93628000$

C $\quad 0.95661100 \quad 3.71233900 \quad-2.15660100$

C $\quad-0.23346400 \quad 4.46768400 \quad-2.28883600$

$\mathrm{H} \quad 1.96010600 \quad 2.09252500 \quad-1.19943100$

C $\quad-1.66169400 \quad 5.88048600 \quad-3.77223300$

$\mathrm{H} \quad-2.27526500 \quad 5.00247100 \quad-4.04421300$

$\mathrm{H} \quad-2.21765500 \quad 6.43489300 \quad-3.00556200$

C $\quad-1.45260300 \quad 6.76432400 \quad-4.98600300$

$\mathrm{H} \quad-0.81718600 \quad 7.62076600 \quad-4.70491600$

$\begin{array}{llll}\mathrm{H} & -0.90127700 & 6.20568600 & -5.76201300\end{array}$

$\begin{array}{llll}\text { C } & -2.76786600 & 7.26929000 & -5.56065300\end{array}$

$\mathrm{H} \quad-3.40183600 \quad 6.40913000 \quad-5.83062200$

$\mathrm{H} \quad-3.31592500 \quad 7.81988300 \quad-4.77906300$

C $\quad-2.56785500 \quad 8.16103400 \quad-6.77422900$

$\mathrm{H} \quad-2.05068000 \quad 7.62314700 \quad-7.58213600$

$\mathrm{H} \quad-3.52384400 \quad 8.52252200 \quad-7.17558600$

$\mathrm{H} \quad-1.95926700 \quad 9.04209900 \quad-6.52324400$

C $\quad-1.23449700 \quad 4.14165300 \quad-1.35861900$

C $\quad-1.15636000 \quad 3.11288200 \quad-0.41035100$

$\begin{array}{llll}\text { O } & -2.41574200 & 4.81866000 & -1.22076400\end{array}$

$\begin{array}{llll}\text { C } & -2.38472500 & 3.19478300 & 0.33667500\end{array}$

C $\quad-3.07993200 \quad 4.22783600 \quad-0.19097200$

$\mathrm{H} \quad-2.69349800 \quad 2.59422800 \quad 1.18424300$

$\mathrm{H} \quad-4.04088900 \quad 4.66460600 \quad 0.05289600$

$\begin{array}{lrrr}\mathrm{C} & 0.01186600 & 2.31443900 & -0.33030200 \\ \mathrm{Cr} & 1.53851500 & 4.51886900 & 1.50483700 \\ \mathrm{C} & 2.00158700 & 3.22219900 & 2.82003400 \\ \mathrm{O} & 2.32505100 & 2.54580400 & 3.69637700 \\ \mathrm{C} & 3.21234600 & 4.23768700 & 0.62587500 \\ \mathrm{O} & 4.24646200 & 4.08009300 & 0.14366400 \\ \mathrm{C} & 2.30796500 & 5.74433000 & 2.59522300 \\ \mathrm{O} & 2.80569000 & 6.52874500 & 3.29619100 \\ \mathrm{C} & -0.12933900 & 4.77136700 & 2.39120200 \\ \mathrm{O} & -1.09357300 & 4.98208900 & 2.98565400 \\ \mathrm{C} & 1.11285400 & 5.96369900 & 0.33188500 \\ \mathrm{O} & 0.89198700 & 6.91971900 & -0.27246300 \\ \mathrm{~N} & -0.37065200 & 5.46218900 & -3.23015200 \\ \mathrm{H} & 0.36509300 & 5.44796700 & -3.93211100 \\ \mathrm{C} & 5.74815300 & 4.61284500 & -5.33020200 \\ \mathrm{H} & 6.60604500 & 4.10262400 & -4.86950000 \\ \mathrm{H} & 5.65162200 & 4.21245500 & -6.34879900 \\ \mathrm{H} & 5.99737300 & 5.67836600 & -5.40738000\end{array}$

\section{TS IX}




$\begin{array}{rrrr}\mathrm{C} & 0.37561300 & 3.14874600 & 1.31008000 \\ \mathrm{H} & 0.51785300 & 2.34017200 & 2.03865800 \\ \mathrm{H} & 1.28404700 & 3.77476500 & 1.34623500 \\ \mathrm{C} & -0.84130300 & 3.97714800 & 1.69821200 \\ \mathrm{H} & -0.98371500 & 4.78691400 & 0.96246900 \\ \mathrm{H} & -1.74733400 & 3.35249100 & 1.64837600 \\ \mathrm{C} & -0.70144800 & 4.56357800 & 3.09482300 \\ \mathrm{H} & -0.57432200 & 3.74566900 & 3.82287400 \\ \mathrm{H} & 0.21983500 & 5.16675200 & 3.14725900 \\ \mathrm{C} & -1.89921600 & 5.41437300 & 3.48140100 \\ \mathrm{H} & -2.82481500 & 4.82076600 & 3.47604300 \\ \mathrm{H} & -1.78592700 & 5.84364200 & 4.48564300 \\ \mathrm{H} & -2.03781000 & 6.24741100 & 2.77701200 \\ \mathrm{C} & 0.19686900 & 3.17705900 & -2.40929900 \\ \mathrm{C} & 0.66655200 & 3.91200200 & -3.47206000 \\ \mathrm{O} & -0.67015700 & 2.22712400 & -2.86211800 \\ \mathrm{C} & 0.07935800 & 3.35994900 & -4.63756100 \\ \mathrm{H} & 1.35804300 & 4.74540500 & -3.40770800 \\ \mathrm{C} & -0.72847400 & 2.34878100 & -4.20079000 \\ \mathrm{H} & 0.22077200 & 3.67291700 & -5.66494800 \\ \mathrm{H} & -1.39914000 & 1.66438100 & -4.70687300 \\ \mathrm{C} & 3.32490800 & -0.82535700 & -7.72657500 \\ \mathrm{H} & 4.32168100 & -0.38951800 & -7.86566700 \\ \mathrm{H} & 3.39845500 & -1.90101000 & -7.94244000 \\ \mathrm{H} & 2.65375900 & -0.39608700 & -8.48258300\end{array}$

IX

$\begin{array}{lrrr}\text { E }=-1749.594019 \mathrm{H} & & \\ \mathrm{Cr} & -1.86063800 & -0.09322100 & 0.02731600 \\ \mathrm{C} & -2.72924400 & 1.54211500 & -0.31903200 \\ \mathrm{C} & -1.90809500 & 0.21446600 & 1.89396100 \\ \mathrm{C} & -3.48960900 & -0.90483500 & 0.22572900 \\ \mathrm{C} & -1.00238900 & -1.78112700 & 0.12964100 \\ \mathrm{C} & -1.98109100 & -0.41987100 & -1.83177800 \\ \mathrm{O} & -0.52786900 & -2.83499600 & 0.10558800 \\ \mathrm{O} & -2.04690900 & 0.37525500 & 3.03292300 \\ \mathrm{O} & -3.33274300 & 2.49464200 & -0.58801100 \\ \mathrm{O} & -2.15909000 & -0.68840900 & -2.94257000 \\ \mathrm{C} & 0.27580300 & 0.84167300 & -0.13046900 \\ \mathrm{C} & 1.38318400 & 0.41248900 & 0.85320400 \\ \mathrm{C} & 2.65851500 & 0.97303500 & 0.66768900 \\ \mathrm{C} & 1.22731400 & -0.50764000 & 1.88864900 \\ \mathrm{C} & 3.72875900 & 0.63366500 & 1.48409400 \\ \mathrm{H} & 2.81209600 & 1.69003100 & -0.14142200 \\ \mathrm{C} & 2.30155500 & -0.86237900 & 2.70711600 \\ \mathrm{H} & 0.25914900 & -0.96295700 & 2.07641400 \\ \mathrm{C} & 3.55416500 & -0.29362800 & 2.51255900 \\ \mathrm{H} & 4.70424300 & 1.08907300 & 1.31526100 \\ \mathrm{H} & 2.14705900 & -1.58910600 & 3.50370100 \\ \mathrm{H} & 4.39089900 & -0.56982900 & 3.15272000 \\ \mathrm{C} & 0.76337600 & 0.43058600 & -1.42517800 \\ \mathrm{C} & 1.88397000 & -0.48639900 & -3.63868700 \\ \mathrm{C} & 1.16432500 & -0.56089000 & -4.84132100 \\ \mathrm{C} & 3.23175800 & -0.88414000 & -3.63957300 \\ \mathrm{C} & 1.77822800 & -1.00991300 & -6.00417700 \\ \mathrm{H} & 0.11455500 & -0.26950500 & -4.85667900 \\ \mathrm{C} & 3.83181000 & -1.33936500 & -4.80564500 \\ \mathrm{H} & 3.79915200 & -0.83635600 & -2.70985400 \\ \mathrm{C} & 3.12049000 & -1.40340000 & -6.00966800\end{array}$

$\begin{array}{lrrr}\mathrm{H} & 1.20290000 & -1.06170900 & -6.92962500 \\ \mathrm{H} & 4.87749200 & -1.64995400 & -4.78644600 \\ \mathrm{O} & -4.52297800 & -1.42509200 & 0.37274100 \\ \mathrm{C} & 1.27745700 & -0.01031100 & -2.43737600 \\ \mathrm{~N} & 0.17388700 & 2.32555800 & -0.02597900 \\ \mathrm{C} & 0.08088400 & 3.20535800 & -0.98925000 \\ \mathrm{H} & 0.24268000 & 4.23911100 & -0.67781700 \\ \mathrm{C} & 0.23766600 & 2.91742600 & 1.34502700 \\ \mathrm{H} & 0.34364500 & 2.09289900 & 2.05439600 \\ \mathrm{H} & 1.16403200 & 3.50825000 & 1.39798700 \\ \mathrm{C} & -0.96624500 & 3.76410800 & 1.71912500 \\ \mathrm{H} & -1.11202700 & 4.58479300 & 0.99882400 \\ \mathrm{H} & -1.88042500 & 3.15233600 & 1.68498700 \\ \mathrm{C} & -0.79646800 & 4.34810000 & 3.11534700 \\ \mathrm{H} & -0.65814800 & 3.53141900 & 3.84188400 \\ \mathrm{H} & 0.12689900 & 4.94878400 & 3.14965400 \\ \mathrm{C} & -1.98534400 & 5.20218300 & 3.52083400 \\ \mathrm{H} & -2.91240800 & 4.61132200 & 3.53111000 \\ \mathrm{H} & -1.85323100 & 5.63034600 & 4.52294900 \\ \mathrm{H} & -2.13247000 & 6.03580500 & 2.81906600 \\ \mathrm{C} & -0.25014400 & 3.10837000 & -2.36743800 \\ \mathrm{C} & -0.06893500 & 4.08134800 & -3.32877100 \\ \mathrm{O} & -0.93958400 & 2.06142800 & -2.90582200 \\ \mathrm{C} & -0.64547400 & 3.58509600 & -4.51848600 \\ \mathrm{H} & 0.42637100 & 5.03427800 & -3.17575600 \\ \mathrm{C} & -1.17163000 & 2.36570700 & -4.19521800 \\ \mathrm{H} & -0.68849300 & 4.06738000 & -5.48725000 \\ \mathrm{H} & -1.74110000 & 1.63848600 & -4.76121500 \\ \mathrm{C} & 3.78710100 & -1.85769400 & -7.27193800 \\ \mathrm{H} & 4.32305800 & -1.02919000 & -7.75816600 \\ \mathrm{H} & 4.52494900 & -2.64707700 & -7.07828800 \\ \mathrm{H} & 3.05828200 & -2.24199800 & -7.99675000\end{array}$

\section{TS X}

$\begin{array}{lrrr}\mathrm{E}=-1749.587722 \mathrm{H} & & \\ \mathrm{Cr} & -1.32478900 & -0.96579700 & -2.03874100 \\ \mathrm{C} & -2.64821400 & 0.38570400 & -2.13976400 \\ \mathrm{C} & -1.89543200 & -1.47304400 & -0.29285000 \\ \mathrm{C} & -2.54687800 & -2.09747400 & -2.75197300 \\ \mathrm{C} & -0.05300800 & -2.38863500 & -2.11263600 \\ \mathrm{C} & -0.79357200 & -0.41533500 & -3.78357500 \\ \mathrm{O} & 0.66546400 & -3.28308200 & -2.21964700 \\ \mathrm{O} & -2.30610800 & -1.82233000 & 0.72642700 \\ \mathrm{O} & -3.50991100 & 1.14040900 & -2.29795800 \\ \mathrm{O} & -0.54033200 & -0.09885600 & -4.86209100 \\ \mathrm{C} & 0.42611300 & 0.79259400 & -0.28603000 \\ \mathrm{C} & 0.87767100 & 0.21379900 & 0.98898500 \\ \mathrm{C} & 0.91454800 & 0.95979400 & 2.17993300 \\ \mathrm{C} & 1.36103600 & -1.10640800 & 1.03428000 \\ \mathrm{C} & 1.37586200 & 0.39868500 & 3.36724900 \\ \mathrm{H} & 0.61536200 & 2.00669600 & 2.17370000 \\ \mathrm{C} & 1.83104000 & -1.66076000 & 2.21736900 \\ \mathrm{H} & 1.36630900 & -1.70740100 & 0.12508000 \\ \mathrm{C} & 1.83283300 & -0.91626200 & 3.39733500 \\ \mathrm{H} & 1.39368900 & 1.00578000 & 4.27189900 \\ \mathrm{H} & 2.19337800 & -2.68813100 & 2.21865700 \\ \mathrm{H} & 2.19845200 & -1.35270200 & 4.32558300 \\ \mathrm{C} & 1.15200500 & 0.47065000 & -1.44600300 \\ \mathrm{C} & 2.72489900 & -0.08808300 & -3.48839800\end{array}$




\begin{tabular}{lrrr} 
C & 2.74053900 & 0.77522200 & -4.60386100 \\
$\mathrm{C}$ & 3.55467600 & -1.22471000 & -3.50749900 \\
$\mathrm{C}$ & 3.54676500 & 0.49198700 & -5.69648900 \\
$\mathrm{H}$ & 2.10817800 & 1.66400300 & -4.60220100 \\
$\mathrm{C}$ & 4.35891100 & -1.48809000 & -4.60786900 \\
$\mathrm{H}$ & 3.55286700 & -1.89843200 & -2.65010700 \\
$\mathrm{C}$ & 4.37048700 & -0.64015800 & -5.72139400 \\
$\mathrm{H}$ & 3.54147200 & 1.16563200 & -6.55521100 \\
$\mathrm{H}$ & 4.99254700 & -2.37614000 & -4.60749200 \\
$\mathrm{O}$ & -3.33389700 & -2.82921600 & -3.20040400 \\
$\mathrm{C}$ & 1.90521700 & 0.19980900 & -2.37519200 \\
$\mathrm{~N}$ & -0.42135900 & 1.90764600 & -0.29366100 \\
$\mathrm{C}$ & -0.40380600 & 2.88590700 & -1.20277500 \\
$\mathrm{H}$ & -1.34886000 & 3.40710800 & -1.35394200 \\
$\mathrm{C}$ & -1.59329400 & 1.90717600 & 0.62150200 \\
$\mathrm{H}$ & -2.48331000 & 1.69506600 & 0.01116800 \\
$\mathrm{H}$ & -1.46890100 & 1.06100100 & 1.30325100 \\
$\mathrm{C}$ & -1.78807200 & 3.19891700 & 1.39519300 \\
$\mathrm{H}$ & -0.82629000 & 3.54803200 & 1.80453300 \\
$\mathrm{H}$ & -2.14508100 & 3.99693900 & 0.72604200 \\
$\mathrm{C}$ & -2.78969600 & 3.00007100 & 2.52425400 \\
$\mathrm{H}$ & -3.72403600 & 2.58105100 & 2.11616100 \\
$\mathrm{H}$ & -2.39609800 & 2.24599900 & 3.22586100 \\
$\mathrm{C}$ & -3.08442500 & 4.29287600 & 3.26427300 \\
$\mathrm{H}$ & -3.51404500 & 5.04674000 & 2.58893300 \\
$\mathrm{H}$ & -3.79704800 & 4.13762400 & 4.08460500 \\
$\mathrm{H}$ & -2.16768600 & 4.72005400 & 3.69588400 \\
$\mathrm{C}$ & 0.66765300 & 3.32784800 & -2.01623100 \\
$\mathrm{C}$ & 0.62735300 & 4.11293200 & -3.15300000 \\
$\mathrm{O}$ & 1.97130000 & 3.12087300 & -1.65784000 \\
$\mathrm{C}$ & 1.97190000 & 4.38806900 & -3.50290900 \\
$\mathrm{H}$ & -0.27322300 & 4.43835000 & -3.66253400 \\
$\mathrm{C}$ & 2.74289900 & 3.76404200 & -2.56524000 \\
$\mathrm{H}$ & 2.32980900 & 4.96830600 & -4.34532800 \\
$\mathrm{H}$ & 3.81250900 & 3.68568500 & -2.41383000 \\
$\mathrm{C}$ & 5.23608000 & -0.92566200 & -6.90982500 \\
$\mathrm{H}$ & 5.99681400 & -0.14449000 & -7.05112500 \\
$\mathrm{H}$ & 5.75896200 & -1.88421200 & -6.80460400 \\
$\mathrm{H}$ & 4.64630700 & -0.96382700 & -7.83645600 \\
& & & \\
\hline
\end{tabular}

$\mathbf{X}$

$\begin{array}{lrrr}\text { E }=-1749.623111 \mathrm{H} & & \\ \mathrm{Cr} & 0.71523900 & -1.02270000 & -3.67631200 \\ \mathrm{C} & -0.14619800 & 0.61328400 & -4.03965300 \\ \mathrm{C} & -0.53541300 & -1.39384700 & -2.30886400 \\ \mathrm{C} & -0.33183800 & -2.05413100 & -4.78196000 \\ \mathrm{C} & 1.82190100 & -2.41861200 & -3.05268100 \\ \mathrm{C} & 1.86233500 & -0.81237000 & -5.16474000 \\ \mathrm{O} & 2.53189700 & -3.23916100 & -2.65019400 \\ \mathrm{O} & -1.35004500 & -1.68177900 & -1.53687100 \\ \mathrm{O} & -0.64376000 & 1.64647900 & -4.20879000 \\ \mathrm{O} & 2.51501800 & -0.75702300 & -6.11942200 \\ \mathrm{C} & 0.91535100 & 0.80215900 & -0.07393000 \\ \mathrm{C} & 1.03436400 & 0.08775000 & 1.20117900 \\ \mathrm{C} & 0.73721300 & 0.71541900 & 2.41877500 \\ \mathrm{C} & 1.47665400 & -1.24417400 & 1.22522300 \\ \mathrm{C} & 0.87617000 & 0.03139800 & 3.62400700 \\ \mathrm{H} & 0.40998400 & 1.75607400 & 2.42459800 \\ \mathrm{C} & 1.62237600 & -1.92068200 & 2.42931900\end{array}$

$\begin{array}{lll}1.70057600 & -1.74882300 & 0.28334200\end{array}$

$1.32085600 \quad-1.28801900 \quad 3.63693400$

$\begin{array}{lll}0.64293900 & 0.53956300 & 4.55918100\end{array}$

$\begin{array}{lll}1.96484200 & -2.95479200 & 2.42542300\end{array}$

$\begin{array}{lll}1.43202200 & -1.82152000 & 4.57980400\end{array}$

$\begin{array}{lll}1.50579700 & 0.47157900 & -1.21979300\end{array}$

$\begin{array}{llll}3.30075600 & 0.69661100 & -2.83948900\end{array}$

$\begin{array}{lll}3.40541700 & 1.72981800 & -3.78030700\end{array}$

$\begin{array}{llll}4.47732900 & 0.13048800 & -2.34035200\end{array}$

$\begin{array}{lll}4.65055300 & 2.18351100 & -4.19804000\end{array}$

$\begin{array}{lll}2.49582400 & 2.17437800 & -4.18950200\end{array}$

$\begin{array}{llll}5.72296800 & 0.58296000 & -2.77557000\end{array}$

$\begin{array}{lll}4.41187900 & -0.67548700 & -1.60707400\end{array}$

$\begin{array}{lll}5.83408000 & 1.61619100 & -3.70793100\end{array}$

$\begin{array}{lll}4.71057800 & 2.99307900 & -4.92847500\end{array}$

$\begin{array}{llll}6.62919400 & 0.12330100 & -2.37738300\end{array}$

$-0.99654900 \quad-2.70556700 \quad-5.48503300$

$\begin{array}{lll}1.98061700 & 0.21277600 & -2.39124000\end{array}$

$\begin{array}{llll}0.03034500 & 1.95340700 & -0.10942100\end{array}$

$\begin{array}{llll}0.39393300 & 3.12636100 & -0.55546200\end{array}$

$\begin{array}{lll}-0.40684100 & 3.84022600 & -0.75388300\end{array}$

$\begin{array}{lll}-1.41235900 & 1.67861200 & 0.12953100\end{array}$

$\begin{array}{lll}-1.88596400 & 1.54775400 & -0.85552200\end{array}$

$\begin{array}{lll}-1.45082000 & 0.70842600 & 0.63663600\end{array}$

$\begin{array}{lll}-2.12555800 & 2.72812400 & 0.95974500\end{array}$

$\begin{array}{lll}-1.53162300 & 2.94536700 & 1.86306200\end{array}$

$\begin{array}{lll}-2.22189000 & 3.67503400 & 0.40593000\end{array}$

$\begin{array}{lll}-3.51322000 & 2.24465700 & 1.36059800\end{array}$

$\begin{array}{lll}-4.08157700 & 1.97267200 & 0.45677900\end{array}$

$\begin{array}{lll}-3.41579100 & 1.32048200 & 1.95204500\end{array}$

$\begin{array}{lll}-4.27494400 & 3.29102500 & 2.15497400\end{array}$

$\begin{array}{lll}-4.42535800 & 4.20713100 & 1.56589900\end{array}$

$\begin{array}{lll}-5.26424300 & 2.92625100 & 2.45995500\end{array}$

$\begin{array}{lll}-3.72860500 & 3.57099100 & 3.06727000\end{array}$

$\begin{array}{lll}1.67951400 & 3.64094800 & -0.83236000\end{array}$

$\begin{array}{lll}1.97526000 & 4.88708600 & -1.35308500\end{array}$

$\begin{array}{llll}2.84125800 & 2.97336700 & -0.56822900\end{array}$

$3.37993100 \quad 4.97568800-1.41612100$

$\begin{array}{llll}1.24601000 & 5.63438500 & -1.64707700\end{array}$

$\begin{array}{llll}3.84694400 & 3.78595900 & -0.92472300\end{array}$

$\begin{array}{llll}3.97913000 & 5.80324700 & -1.77483800\end{array}$

$\begin{array}{llll}4.84608900 & 3.39619500 & -0.76835800\end{array}$

$\begin{array}{llll}7.16949700 & 2.10265300 & -4.18647700\end{array}$

$\begin{array}{llll}7.28880500 & 3.18335600 & -4.02426000\end{array}$

$\begin{array}{lll}7.99136100 & 1.59341800 & -3.66730900\end{array}$

$\begin{array}{llll}7.29894600 & 1.92758900 & -5.26405100\end{array}$

\section{TS XI}

$\begin{array}{lrrr}\text { E }=-1749.592323 \mathrm{H} & & \\ \mathrm{Cr} & -0.84534100 & 0.32218900 & -2.88559500 \\ \mathrm{C} & -2.19044600 & 0.12934000 & -1.55998600 \\ \mathrm{C} & -0.19352600 & -1.38469500 & -2.36395400 \\ \mathrm{C} & -2.10067600 & -0.39458800 & -3.98798600 \\ \mathrm{C} & 0.20504100 & 0.35794400 & -4.46645500 \\ \mathrm{C} & -1.44795800 & 2.09757800 & -3.18705000 \\ \mathrm{O} & 0.70138700 & 0.32457000 & -5.50940600 \\ \mathrm{O} & 0.16560500 & -2.42711500 & -2.02286600 \\ \mathrm{O} & -3.12065300 & -0.08132500 & -0.90747700 \\ \mathrm{O} & -1.84171900 & 3.17021500 & -3.35173800\end{array}$




\begin{tabular}{|c|c|c|c|}
\hline 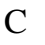 & 0.47589600 & 1.32647200 & -0.35176300 \\
\hline C & -0.37735200 & 0.96186700 & 0.73021500 \\
\hline C & -1.35378800 & 1.85856600 & 1.19508000 \\
\hline $\mathrm{C}$ & -0.23181100 & -0.27831900 & 1.37759100 \\
\hline C & -2.16042100 & 1.51753200 & 2.27370700 \\
\hline $\mathrm{H}$ & -1.49391000 & 2.81259000 & 0.68448300 \\
\hline C & -1.05168400 & -0.60800300 & 2.44614600 \\
\hline $\mathrm{H}$ & 0.52626600 & -0.97805000 & 1.02494400 \\
\hline $\mathrm{C}$ & -2.02536600 & 0.28397000 & 2.91755700 \\
\hline $\mathrm{H}$ & -2.91903000 & 2.21996700 & 2.62119900 \\
\hline $\mathrm{H}$ & -0.93686100 & -1.57857000 & 2.93143100 \\
\hline C & 0.89467500 & 1.35431400 & -1.54913700 \\
\hline C & 2.96103300 & 1.35916200 & -2.97487000 \\
\hline C & 3.05060800 & -0.04212700 & -3.04535000 \\
\hline C & 4.09628700 & 2.10509500 & -3.34471800 \\
\hline $\mathrm{C}$ & 4.20628900 & -0.66711700 & -3.49450900 \\
\hline $\mathrm{H}$ & 2.19564600 & -0.64943800 & -2.75463800 \\
\hline $\mathrm{C}$ & 5.24826400 & 1.47707800 & -3.80376400 \\
\hline $\mathrm{H}$ & 4.09524000 & 3.18822600 & -3.23177500 \\
\hline C & 5.31093300 & 0.08748800 & -3.88783000 \\
\hline $\mathrm{H}$ & 4.24099300 & -1.75468800 & -3.54269900 \\
\hline $\mathrm{H}$ & 6.11192900 & 2.08116300 & -4.07941700 \\
\hline $\mathrm{O}$ & -2.89757700 & -0.85419100 & -4.70255100 \\
\hline C & 1.77525400 & 2.02021500 & -2.43039000 \\
\hline $\mathrm{N}$ & 1.53429300 & 3.35502000 & -2.68880700 \\
\hline $\mathrm{C}$ & 0.99139600 & 4.18707300 & -1.75805500 \\
\hline $\mathrm{H}$ & 0.42637700 & 5.04001300 & -2.13097900 \\
\hline C & 1.62945500 & 3.88130400 & -4.07142700 \\
\hline $\mathrm{H}$ & 2.11358300 & 3.10811300 & -4.67702300 \\
\hline $\mathrm{H}$ & 0.59937700 & 3.98562900 & -4.44900700 \\
\hline C & 2.37226300 & 5.19868800 & -4.19118100 \\
\hline $\mathrm{H}$ & 1.78797700 & 6.01219600 & -3.73396500 \\
\hline $\mathrm{H}$ & 3.32070900 & 5.14762400 & -3.63174200 \\
\hline C & 2.65495900 & 5.53296600 & -5.64921200 \\
\hline $\mathrm{H}$ & 3.28503700 & 4.73985200 & -6.08446700 \\
\hline $\mathrm{H}$ & 1.71121500 & 5.52155500 & -6.21827400 \\
\hline $\mathrm{C}$ & 3.33720700 & 6.88073100 & -5.80645000 \\
\hline $\mathrm{H}$ & 4.28722800 & 6.90953400 & -5.25322000 \\
\hline $\mathrm{H}$ & 3.55789500 & 7.10477200 & -6.85821300 \\
\hline $\mathrm{H}$ & 2.70531800 & 7.69259200 & -5.41865500 \\
\hline $\mathrm{C}$ & 1.10027000 & 3.99721700 & -0.39150600 \\
\hline $\mathrm{C}$ & 1.78863100 & 3.06807600 & 0.41797900 \\
\hline $\mathrm{O}$ & 0.38886900 & 4.85345200 & 0.41959200 \\
\hline $\mathrm{C}$ & 1.53986000 & 3.47162000 & 1.76550200 \\
\hline $\mathrm{H}$ & 2.60729100 & 2.44020900 & 0.08320600 \\
\hline C & 0.67502600 & 4.51390900 & 1.70382600 \\
\hline $\mathrm{H}$ & 1.92598400 & 3.01091900 & 2.66736200 \\
\hline $\mathrm{H}$ & 0.18573400 & 5.11024400 & 2.46465700 \\
\hline C & -2.88322000 & -0.08123900 & 4.08935200 \\
\hline $\mathrm{H}$ & -3.34572400 & -1.06886200 & 3.95672400 \\
\hline $\mathrm{H}$ & -2.29211600 & -0.12985500 & 5.01543900 \\
\hline $\mathrm{H}$ & -3.68509300 & 0.65075900 & 4.24526800 \\
\hline $\mathrm{H}$ & 6.21490900 & -0.40273900 & -4.24574200 \\
\hline
\end{tabular}

\section{XI}

$\mathrm{E}=-1749.630030 \mathrm{H}$

$\begin{array}{cccc}\mathrm{Cr} & -0.87918400 & 0.40267400 & -2.82497100 \\ \mathrm{C} & -2.14679000 & 0.30653300 & -1.41194900 \\ \mathrm{C} & -0.23933700 & -1.29546500 & -2.27757800\end{array}$ $\begin{array}{lll}-2.21615200 & -0.36699200 & -3.81243000\end{array}$

$\begin{array}{llll}0.06442600 & 0.38537900 & -4.46405300\end{array}$

$\begin{array}{lll}-1.55983400 & 2.12870000 & -3.20576400\end{array}$

$\begin{array}{llll}0.50081700 & 0.32335900 & -5.53543800\end{array}$

$\begin{array}{lll}0.09290400 & -2.34732600 & -1.93274000\end{array}$

$\begin{array}{llll}-3.05894800 & 0.18502100 & -0.71369400\end{array}$

$\begin{array}{lll}-2.04909600 & 3.14876300 & -3.44401800\end{array}$

$\begin{array}{llll}0.62025400 & 1.76088900 & -0.23083200\end{array}$

$\begin{array}{lll}-0.19811000 & 1.09792300 & 0.80455700\end{array}$

$\begin{array}{lll}-1.19185000 & 1.77537700 & 1.52470600\end{array}$

$\begin{array}{lll}0.03247700 & -0.24772000 & 1.10907900\end{array}$

$\begin{array}{lll}-1.92255700 & 1.12520100 & 2.51108700\end{array}$

$\begin{array}{lll}-1.41863700 & 2.81568500 & 1.28811800\end{array}$

$\begin{array}{lll}-0.70221900 & -0.89564800 & 2.09916300\end{array}$

$\begin{array}{lll}0.80991200 & -0.78701600 & 0.56628400\end{array}$

$\begin{array}{lll}-1.68685600 & -0.21928500 & 2.82247700\end{array}$

$\begin{array}{lll}-2.70210500 & 1.66776300 & 3.04895900\end{array}$

$\begin{array}{lll}-0.50189300 & -1.94571800 & 2.31803200\end{array}$

$\begin{array}{lll}0.68328000 & 1.42255800 & -1.52653000\end{array}$

$\begin{array}{lll}2.75940900 & 1.40027000 & -2.95115500\end{array}$

$\begin{array}{lll}2.85281000 & 0.00609700 & -3.06143900\end{array}$

$3.93146400 \quad 2.15515800-3.13719000$

$4.05461600 \quad-0.60859800-3.38703100$

$1.96912200 \quad-0.60288400-2.90174500$

$\begin{array}{llll}5.13436700 & 1.53999000 & -3.46301500\end{array}$

$3.91540500 \quad 3.23273500-2.98311000$

$\begin{array}{lll}5.20011900 & 0.15532000 & -3.59948000\end{array}$

$\begin{array}{lll}4.09319300 & -1.69283500 & -3.47738900\end{array}$

$\begin{array}{lll}6.02804200 & 2.14748300 & -3.59643400\end{array}$

$\begin{array}{lll}-3.06088800 & -0.85998200 & -4.44473100\end{array}$

$\begin{array}{lll}1.53956900 & 2.08314200 & -2.46478800\end{array}$

$\begin{array}{llll}1.32888200 & 3.37371500 & -2.81476000\end{array}$

$\begin{array}{llll}0.72131500 & 4.28957100 & -1.94234200\end{array}$

$\begin{array}{lll}0.21385400 & 5.13587100 & -2.39877100\end{array}$

$\begin{array}{lll}1.58325000 & 3.87275400 & -4.18430900\end{array}$

$\begin{array}{llll}2.09851200 & 3.07717400 & -4.73296900\end{array}$

$\begin{array}{lll}0.59769800 & 4.00015500 & -4.66134800\end{array}$

$\begin{array}{lll}2.37051100 & 5.17081800 & -4.24906200\end{array}$

$\begin{array}{lll}1.73346600 & 6.02050000 & -3.95995300\end{array}$

$3.20062000 \quad 5.15090400-3.52460400$

$\begin{array}{llll}2.91777600 & 5.40066100 & -5.65117800\end{array}$

$3.62256200 \quad 4.58935000 \quad-5.89794100$

$\begin{array}{lll}2.09641200 & 5.32438300 & -6.38230200\end{array}$

$3.60667800 \quad 6.74695100 \quad-5.78768500$

$\begin{array}{llll}4.43150200 & 6.84514900 & -5.06690500\end{array}$

$\begin{array}{llll}4.02576800 & 6.88846500 & -6.79241100\end{array}$

$\begin{array}{llll}2.90542100 & 7.57258600 & -5.60009000\end{array}$

$\begin{array}{lll}0.84547500 & 4.16261200 & -0.60856300\end{array}$

$\begin{array}{llll}1.45562200 & 3.02088600 & 0.14799000\end{array}$

$\begin{array}{lll}0.34311900 & 5.09637900 & 0.25087400\end{array}$

$\begin{array}{lll}1.37349700 & 3.54365600 & 1.54903900\end{array}$

$\begin{array}{lll}2.49397500 & 2.83378700 & -0.17945500\end{array}$

$\begin{array}{lll}0.72596200 & 4.70721900 & 1.52256900\end{array}$

$\begin{array}{lll}1.74960300 & 3.04213500 & 2.43348900\end{array}$

$\begin{array}{lll}0.43537600 & 5.39795900 & 2.30568200\end{array}$

$\begin{array}{lll}6.14143900 & -0.32742300 & -3.85728400\end{array}$

$\begin{array}{lll}-2.45860100 & -0.89871200 & 3.91300000\end{array}$

$\begin{array}{lll}-2.30905800 & -1.98545400 & 3.89432500\end{array}$

$\begin{array}{lll}-2.14696100 & -0.53988500 & 4.90474700\end{array}$

$\begin{array}{lll}-3.53588400 & -0.70224100 & 3.82771100\end{array}$ 


\section{TS XII}

\begin{tabular}{|c|c|c|c|}
\hline \multicolumn{4}{|c|}{$E=-1749.591639 H$} \\
\hline $\mathrm{Cr}$ & -0.99862700 & 0.34113900 & -2.84688700 \\
\hline $\mathrm{C}$ & -2.24416300 & 0.28095900 & -1.42021900 \\
\hline $\mathrm{C}$ & -0.28375100 & -1.30957000 & -2.27518500 \\
\hline $\mathrm{C}$ & -2.31467400 & -0.47920500 & -3.83215500 \\
\hline $\mathrm{C}$ & 0.00061700 & 0.28549700 & -4.44938700 \\
\hline $\mathrm{C}$ & -1.67788200 & 2.05897400 & -3.22856500 \\
\hline $\mathrm{O}$ & 0.48579300 & 0.18067100 & -5.49758000 \\
\hline $\mathrm{O}$ & 0.12582000 & -2.33263100 & -1.91995200 \\
\hline $\mathrm{O}$ & -3.13132200 & 0.20476300 & -0.68126900 \\
\hline $\mathrm{O}$ & -2.14676800 & 3.09778000 & -3.43756000 \\
\hline $\mathrm{C}$ & 0.54708900 & 1.69392300 & -0.37317200 \\
\hline $\mathrm{C}$ & -0.24040300 & 1.12418700 & 0.73482400 \\
\hline $\mathrm{C}$ & -1.06763900 & 1.91873600 & 1.53844400 \\
\hline $\mathrm{C}$ & -0.15222500 & -0.24189800 & 1.03072500 \\
\hline $\mathrm{C}$ & -1.78743700 & 1.36260300 & 2.59052600 \\
\hline $\mathrm{H}$ & -1.16743100 & 2.98503200 & 1.32573100 \\
\hline $\mathrm{C}$ & -0.87127000 & -0.79404400 & 2.08493600 \\
\hline $\mathrm{H}$ & 0.50047700 & -0.87295700 & 0.42666900 \\
\hline $\mathrm{C}$ & -1.70060600 & -0.00205100 & 2.88530200 \\
\hline $\mathrm{H}$ & -2.43622900 & 1.99851000 & 3.19513500 \\
\hline $\mathrm{H}$ & -0.78654200 & -1.86186500 & 2.29376700 \\
\hline $\mathrm{C}$ & 0.54786600 & 1.36259900 & -1.68102600 \\
\hline $\mathrm{C}$ & 2.76328700 & 1.45702500 & -3.03393600 \\
\hline $\mathrm{C}$ & 2.88631200 & 0.06364800 & -2.95958200 \\
\hline $\mathrm{C}$ & 3.82925000 & 2.18994700 & -3.58044400 \\
\hline $\mathrm{C}$ & 4.01292200 & -0.58049200 & -3.45857700 \\
\hline $\mathrm{H}$ & 2.08581200 & -0.51720200 & -2.50873900 \\
\hline $\mathrm{C}$ & 4.95040800 & 1.54640500 & -4.08854500 \\
\hline $\mathrm{H}$ & 3.79527500 & 3.27874900 & -3.58398200 \\
\hline $\mathrm{C}$ & 5.04394600 & 0.15590700 & -4.03677200 \\
\hline $\mathrm{H}$ & 4.08152700 & -1.66553700 & -3.39814700 \\
\hline $\mathrm{H}$ & 5.76271400 & 2.13447900 & -4.51300100 \\
\hline $\mathrm{O}$ & -3.14628400 & -1.00201900 & -4.46085800 \\
\hline $\mathrm{C}$ & 1.61356200 & 2.14182900 & -2.39948100 \\
\hline $\mathrm{N}$ & 1.20312600 & 3.38506800 & -2.91432300 \\
\hline $\mathrm{C}$ & 0.75401100 & 4.34892600 & -2.06367100 \\
\hline $\mathrm{H}$ & 0.41282600 & 5.28412400 & -2.49940700 \\
\hline $\mathrm{C}$ & 1.22929500 & 3.71421300 & -4.36078200 \\
\hline $\mathrm{H}$ & 1.70004400 & 2.86921000 & -4.87291500 \\
\hline $\mathrm{H}$ & 0.18294100 & 3.74813000 & -4.69889700 \\
\hline $\mathrm{C}$ & 1.94050000 & 5.01189000 & -4.70831600 \\
\hline $\mathrm{H}$ & 1.33718900 & 5.88008200 & -4.40101100 \\
\hline $\mathrm{H}$ & 2.89169900 & 5.08207200 & -4.15616700 \\
\hline $\mathrm{C}$ & 2.20781800 & 5.08947800 & -6.20513000 \\
\hline $\mathrm{H}$ & 2.85231500 & 4.24435300 & -6.49803000 \\
\hline $\mathrm{H}$ & 1.26053200 & 4.95621200 & -6.75218200 \\
\hline $\mathrm{C}$ & 2.85813300 & 6.40126600 & -6.60743400 \\
\hline $\mathrm{H}$ & 3.81942800 & 6.54246300 & -6.09222000 \\
\hline $\mathrm{H}$ & 3.05268400 & 6.44081500 & -7.68695200 \\
\hline $\mathrm{H}$ & 2.21811400 & 7.25832500 & -6.35333200 \\
\hline $\mathrm{C}$ & 0.91095300 & 4.17596600 & -0.71649100 \\
\hline $\mathrm{C}$ & 1.37133300 & 2.94764400 & -0.10384600 \\
\hline $\mathrm{O}$ & 0.94784200 & 5.21394300 & 0.16600800 \\
\hline $\mathrm{C}$ & 1.80609400 & 3.39755800 & 1.21328800 \\
\hline $\mathrm{H}$ & 2.16590400 & 2.61269700 & -1.13798000 \\
\hline $\mathrm{C}$ & 1.51467100 & 4.71107700 & 1.30276200 \\
\hline
\end{tabular}

$\begin{array}{lll}2.21026700 & 2.77828600 & 2.00593900\end{array}$

$\begin{array}{lll}1.63292000 & 5.43019800 & 2.10531000\end{array}$

$\begin{array}{lll}5.92315200 & -0.34864500 & -4.43443800\end{array}$

$-2.44881700 \quad-0.59495600 \quad 4.04050800$

$\begin{array}{lll}-2.77030600 & -1.62248200 & 3.82631800\end{array}$

$\begin{array}{lll}-1.82183700 & -0.63575500 & 4.94352400\end{array}$

$\begin{array}{lll}-3.33910200 & -0.00437100 & 4.29169300\end{array}$

\section{XII}

\begin{tabular}{|c|c|c|c|}
\hline \multicolumn{4}{|c|}{$E=-1749.646381 H$} \\
\hline $\mathrm{Cr}$ & -0.41034800 & 0.50726600 & -2.75434600 \\
\hline $\mathrm{C}$ & -1.81710500 & -0.03236200 & -1.61154400 \\
\hline $\mathrm{C}$ & 0.39465300 & -1.15107600 & -2.35203900 \\
\hline $\mathrm{C}$ & -1.51730400 & -0.17674200 & -4.05484100 \\
\hline $\mathrm{C}$ & 0.72453200 & 0.72793800 & -4.24846300 \\
\hline $\mathrm{C}$ & -1.18735100 & 2.22121600 & -2.78167600 \\
\hline $\mathrm{O}$ & 1.26730200 & 0.73925900 & -5.27454500 \\
\hline $\mathrm{O}$ & 0.82572600 & -2.19369300 & -2.09046500 \\
\hline $\mathrm{O}$ & -2.77365800 & -0.46173800 & -1.11887300 \\
\hline $\mathrm{O}$ & -1.68728800 & 3.26472500 & -2.70416700 \\
\hline $\mathrm{C}$ & 0.50636800 & 1.93856500 & -0.06480900 \\
\hline $\mathrm{C}$ & -0.52416800 & 1.19680800 & 0.71498700 \\
\hline $\mathrm{C}$ & -1.71265800 & 1.80834000 & 1.12609200 \\
\hline $\mathrm{C}$ & -0.28590400 & -0.12222200 & 1.11952300 \\
\hline $\mathrm{C}$ & -2.64124100 & 1.11648800 & 1.89836400 \\
\hline $\mathrm{H}$ & -1.91760000 & 2.83930600 & 0.83050200 \\
\hline $\mathrm{C}$ & -1.21239200 & -0.80857600 & 1.89607600 \\
\hline $\mathrm{H}$ & 0.64674900 & -0.60808200 & 0.82735600 \\
\hline $\mathrm{C}$ & -2.40942200 & -0.20373500 & 2.29400600 \\
\hline $\mathrm{H}$ & -3.56776100 & 1.60928400 & 2.19777500 \\
\hline $\mathrm{H}$ & -1.00634300 & -1.83659000 & 2.19974000 \\
\hline $\mathrm{C}$ & 0.88344200 & 1.57085600 & -1.34460900 \\
\hline $\mathrm{C}$ & 3.15859600 & 1.49704200 & -2.63361400 \\
\hline $\mathrm{C}$ & 3.29570300 & 0.12755700 & -2.39306600 \\
\hline $\mathrm{C}$ & 4.04668100 & 2.10233700 & -3.52623800 \\
\hline $\mathrm{C}$ & 4.23433700 & -0.63315200 & -3.08211300 \\
\hline $\mathrm{H}$ & 2.66433900 & -0.34646600 & -1.64383700 \\
\hline $\mathrm{C}$ & 4.98525500 & 1.34406000 & -4.22347700 \\
\hline $\mathrm{H}$ & 4.02479400 & 3.17884200 & -3.68850600 \\
\hline $\mathrm{C}$ & 5.07290500 & -0.02982800 & -4.01726400 \\
\hline $\mathrm{H}$ & 4.31096800 & -1.70174200 & -2.88347100 \\
\hline $\mathrm{H}$ & 5.65471100 & 1.83599000 & -4.92789400 \\
\hline $\mathrm{O}$ & -2.21059900 & -0.59355700 & -4.89413800 \\
\hline $\mathrm{C}$ & 2.18299900 & 2.26722000 & -1.77563900 \\
\hline $\mathrm{N}$ & 1.83908500 & 3.62091100 & -2.27472600 \\
\hline $\mathrm{C}$ & 1.65710000 & 4.56700400 & -1.36983000 \\
\hline $\mathrm{H}$ & 1.54631400 & 5.59390100 & -1.71784400 \\
\hline $\mathrm{C}$ & 1.54722500 & 3.91786600 & -3.69545200 \\
\hline $\mathrm{H}$ & 1.84850900 & 3.04107300 & -4.27368500 \\
\hline $\mathrm{H}$ & 0.46004500 & 4.02306500 & -3.81133600 \\
\hline $\mathrm{C}$ & 2.25474600 & 5.15631600 & -4.22485000 \\
\hline $\mathrm{H}$ & 1.80185800 & 6.07155900 & -3.81350200 \\
\hline $\mathrm{H}$ & 3.30943500 & 5.15700600 & -3.90488300 \\
\hline $\mathrm{C}$ & 2.17867900 & 5.19935200 & -5.74526600 \\
\hline $\mathrm{H}$ & 2.65751100 & 4.29439100 & -6.15472900 \\
\hline $\mathrm{H}$ & 1.12376700 & 5.15712600 & -6.06001400 \\
\hline $\mathrm{C}$ & 2.84227200 & 6.44039200 & -6.31525000 \\
\hline $\mathrm{H}$ & 3.90388900 & 6.48613800 & -6.03240800 \\
\hline $\mathrm{H}$ & 2.79019700 & 6.45907100 & -7.41140200 \\
\hline
\end{tabular}




$\begin{array}{lrrr}\mathrm{H} & 2.36051200 & 7.35598100 & -5.94326200 \\ \mathrm{C} & 1.53516200 & 4.27063200 & -0.01486400 \\ \mathrm{C} & 1.01043400 & 3.11785500 & 0.60439100 \\ \mathrm{O} & 1.67496300 & 5.28222900 & 0.89933100 \\ \mathrm{C} & 0.85081400 & 3.49527200 & 1.97638000 \\ \mathrm{H} & 2.76378900 & 2.48813000 & -0.86426200 \\ \mathrm{C} & 1.27719200 & 4.77928700 & 2.08597500 \\ \mathrm{H} & 0.47102600 & 2.87676100 & 2.78122000 \\ \mathrm{H} & 1.36118000 & 5.45107700 & 2.93211600 \\ \mathrm{H} & 5.80188200 & -0.62309700 & -4.56726000 \\ \mathrm{C} & -3.41742300 & -0.96243900 & 3.10251700 \\ \mathrm{H} & -3.94182800 & -1.70584000 & 2.48462700 \\ \mathrm{H} & -2.94364900 & -1.51141800 & 3.92760800 \\ \mathrm{H} & -4.17652600 & -0.29474400 & 3.52909400\end{array}$

\section{TSaze}

$\begin{array}{lrrr}\mathrm{E}=-1749.585272 \mathrm{H} & & \\ \mathrm{Cr} & -1.53497100 & -0.03389200 & -3.02462600 \\ \mathrm{C} & -2.95779400 & -0.35295000 & -1.82617100 \\ \mathrm{C} & -0.67530600 & -1.57183600 & -2.33896100 \\ \mathrm{C} & -2.58060900 & -0.92565100 & -4.26141100 \\ \mathrm{C} & -0.26904800 & 0.16464400 & -4.41783800 \\ \mathrm{C} & -2.20018800 & 1.69004200 & -3.40686600 \\ \mathrm{O} & 0.42056800 & 0.25966400 & -5.34292800 \\ \mathrm{O} & -0.15107400 & -2.48391600 & -1.85712900 \\ \mathrm{O} & -3.88206200 & -0.61785500 & -1.18143600 \\ \mathrm{O} & -2.56107400 & 2.77221400 & -3.60187800 \\ \mathrm{C} & -0.33378800 & 1.00722600 & -1.56757300 \\ \mathrm{C} & -0.69484400 & 1.13047700 & -0.14140000 \\ \mathrm{C} & -1.90898500 & 1.68963400 & 0.27576800 \\ \mathrm{C} & 0.21084700 & 0.67817500 & 0.83031900 \\ \mathrm{C} & -2.20182400 & 1.80862500 & 1.63037900 \\ \mathrm{H} & -2.61960800 & 2.05468400 & -0.46667400 \\ \mathrm{C} & -0.09863100 & 0.76864900 & 2.18417400 \\ \mathrm{H} & 1.16009900 & 0.24325700 & 0.51017800 \\ \mathrm{C} & -1.30304800 & 1.34111900 & 2.58915300 \\ \mathrm{H} & -3.14421700 & 2.25912300 & 1.93995200 \\ \mathrm{H} & 0.60941800 & 0.39732300 & 2.92473300 \\ \mathrm{H} & -1.54446200 & 1.41796300 & 3.64857200 \\ \mathrm{C} & 0.78439200 & 1.56261200 & -1.98108200 \\ \mathrm{C} & 3.11076100 & 0.99757700 & -2.82318100 \\ \mathrm{C} & 2.97738000 & -0.39508500 & -2.72150200 \\ \mathrm{C} & 4.35972400 & 1.52744100 & -3.18034100 \\ \mathrm{C} & 4.05023500 & -1.22858600 & -2.99919700 \\ \mathrm{H} & 2.02053600 & -0.82231800 & -2.42013700 \\ \mathrm{C} & 5.42760800 & 0.68457300 & -3.45840000 \\ \mathrm{H} & 4.49382600 & 2.60884900 & -3.22583000 \\ \mathrm{C} & 5.29271500 & -0.70667100 & -3.37997800 \\ \mathrm{H} & 3.92731400 & -2.30891800 & -2.91527700 \\ \mathrm{H} & 6.39235800 & 1.11250200 & -3.73430500 \\ \mathrm{O} & -3.24244700 & -1.48733100 & -5.03660700 \\ \mathrm{C} & 1.97494800 & 1.86907600 & -2.56754500 \\ \mathrm{~N} & 2.07794600 & 3.27873200 & -2.65272800 \\ \mathrm{C} & 1.19894100 & 3.60539900 & -1.62447200 \\ \mathrm{H} & 0.28105800 & 4.15228100 & -1.87974200 \\ \mathrm{C} & 1.88582500 & 3.92810100 & -3.95466800 \\ \mathrm{H} & 2.43557500 & 3.33298100 & -4.69676100 \\ \mathrm{H} & 0.81215400 & 3.86875400 & -4.22104500 \\ \mathrm{C} & 2.36268000 & 5.36528300 & -3.944419600\end{array}$

$\begin{array}{lll}1.84590500 & 5.92126700 & -3.14271100\end{array}$

$3.43667800 \quad 5.38597400-3.69429700$

$2.12213300 \quad 6.05535200-5.27811300$

$\begin{array}{lll}2.63250000 & 5.49225000 & -6.07618900\end{array}$

$\begin{array}{lll}1.04727800 & 6.01923800 & -5.51827100\end{array}$

$2.60244700 \quad 7.49642300-5.27513000$

$3.67955700 \quad 7.55442200 \quad-5.06126200$

$\begin{array}{llll}2.43019300 & 7.98563700 & -6.24269400\end{array}$

$\begin{array}{llll}2.08193400 & 8.08595700 & -4.50651100\end{array}$

$\begin{array}{llll}1.64057200 & 3.70128300 & -0.27476100\end{array}$

$\begin{array}{llll}2.84395600 & 3.49456800 & 0.36157600\end{array}$

$\begin{array}{lll}0.69525300 & 4.07552200 & 0.63922500\end{array}$

$\begin{array}{llll}2.61650500 & 3.75277600 & 1.73381000\end{array}$

$\begin{array}{lll}3.77211200 & 3.19886400 & -0.11622800\end{array}$

$\begin{array}{lll}1.29646600 & 4.09438300 & 1.84308600\end{array}$

$\begin{array}{lll}3.33571000 & 3.70140900 & 2.54248600\end{array}$

$\begin{array}{lll}0.67445700 & 4.38397400 & 2.68146300\end{array}$

$\begin{array}{lll}6.44183600 & -1.60821300 & -3.70728100\end{array}$

$6.53530000 \quad-1.74790100-4.79429900$

$\begin{array}{lll}7.39512000 & -1.19306400 & -3.35486700\end{array}$

$\begin{array}{lll}6.31436800 & -2.60241800 & -3.26119700\end{array}$

\section{6b}

$\begin{array}{lrrr}\mathrm{E}=-1749.640835 \mathrm{H} & & \\ \mathrm{Cr} & -2.44684900 & 0.99239500 & -0.59110900 \\ \mathrm{C} & -2.17968000 & 2.62710900 & 0.32136600 \\ \mathrm{C} & -2.26722700 & 0.02581600 & 1.02630200 \\ \mathrm{C} & -4.26556300 & 0.91576500 & -0.26000400 \\ \mathrm{C} & -2.37853800 & -0.63436400 & -1.53776300 \\ \mathrm{C} & -2.96716000 & 1.86136900 & -2.17794800 \\ \mathrm{O} & -2.31767400 & -1.63213000 & -2.12164400 \\ \mathrm{O} & -2.27953600 & -0.58262800 & 2.01043100 \\ \mathrm{O} & -1.98371600 & 3.61201700 & 0.89418700 \\ \mathrm{O} & -3.44381500 & 2.33574600 & -3.12399000 \\ \mathrm{C} & -0.34182800 & 1.05692700 & -0.92754000 \\ \mathrm{C} & 0.47302400 & 0.11125200 & -0.13752500 \\ \mathrm{C} & 0.80593100 & 0.40594700 & 1.19199200 \\ \mathrm{C} & 0.89419500 & -1.10914600 & -0.68079600 \\ \mathrm{C} & 1.55820600 & -0.48614700 & 1.94879600 \\ \mathrm{H} & 0.46886900 & 1.34863600 & 1.62914000 \\ \mathrm{C} & 1.63359900 & -2.00836100 & 0.08421900 \\ \mathrm{H} & 0.63904200 & -1.35026400 & -1.71421900 \\ \mathrm{C} & 1.97347300 & -1.69990200 & 1.40000700 \\ \mathrm{H} & 1.81500300 & -0.23568600 & 2.97795200 \\ \mathrm{H} & 1.95072100 & -2.95414100 & -0.35461200 \\ \mathrm{H} & 2.55309900 & -2.40254600 & 1.99726600 \\ \mathrm{C} & 0.31541400 & 1.83998300 & -1.81894400 \\ \mathrm{C} & 2.96805300 & 1.36298100 & -2.17385700 \\ \mathrm{C} & 3.73052300 & 0.97450300 & -3.28606800 \\ \mathrm{C} & 3.48638900 & 1.16524800 & -0.88903200 \\ \mathrm{C} & 4.97526100 & 0.39060100 & -3.10736300 \\ \mathrm{H} & 3.33251800 & 1.09346200 & -4.29312500 \\ \mathrm{C} & 4.74338200 & 0.59921900 & -0.72372700 \\ \mathrm{H} & 2.90411500 & 1.46450500 & -0.01868300 \\ \mathrm{C} & 5.50498700 & 0.19568000 & -1.82509400 \\ \mathrm{H} & 5.55203400 & 0.07636400 & -3.97801000 \\ \mathrm{H} & 5.14097800 & 0.46050700 & 0.28209400 \\ \mathrm{O} & -5.41129100 & 0.87265500 & -0.05799700 \\ \mathrm{C} & 1.65869400 & 1.96808800 & -2.35873300\end{array}$




$\begin{array}{lrrr}\mathrm{N} & 1.31648300 & 2.88279000 & -3.26120600 \\ \mathrm{C} & -0.11139400 & 2.91555500 & -2.82688100 \\ \mathrm{H} & -0.76316200 & 2.56898100 & -3.63914000 \\ \mathrm{C} & 2.02120200 & 3.73789200 & -4.20031200 \\ \mathrm{H} & 2.64295500 & 3.11664500 & -4.85814000 \\ \mathrm{H} & 1.24755800 & 4.20037400 & -4.82862300 \\ \mathrm{C} & 2.86386200 & 4.80274400 & -3.51138300 \\ \mathrm{H} & 2.21222900 & 5.44648000 & -2.89851100 \\ \mathrm{H} & 3.57009700 & 4.31559000 & -2.81924600 \\ \mathrm{C} & 3.62499500 & 5.64604400 & -4.52279800 \\ \mathrm{H} & 4.27204400 & 4.99146900 & -5.12900600 \\ \mathrm{H} & 2.91010800 & 6.10571300 & -5.22433800 \\ \mathrm{C} & 4.46070100 & 6.72578000 & -3.85691800 \\ \mathrm{H} & 5.20240300 & 6.28884200 & -3.17280100 \\ \mathrm{H} & 5.00456700 & 7.33048400 & -4.59418800 \\ \mathrm{H} & 3.83043500 & 7.40676100 & -3.26704800 \\ \mathrm{C} & -0.56056000 & 4.23670400 & -2.35027700 \\ \mathrm{C} & -1.50936300 & 5.10385200 & -2.80105100 \\ \mathrm{O} & 0.08858600 & 4.77270800 & -1.27742400 \\ \mathrm{C} & -1.44744500 & 6.24852700 & -1.94817000 \\ \mathrm{H} & -2.18078100 & 4.93371300 & -3.63610300 \\ \mathrm{C} & -0.46262200 & 5.98954400 & -1.04767400 \\ \mathrm{H} & -2.06035600 & 7.14105500 & -1.99345900 \\ \mathrm{H} & -0.05462100 & 6.54147300 & -0.20996300 \\ \mathrm{C} & 6.84028900 & -0.45522700 & -1.64907700 \\ \mathrm{H} & 6.77191000 & -1.53857200 & -1.82486700 \\ \mathrm{H} & 7.57471600 & -0.06230600 & -2.36446700 \\ \mathrm{H} & 7.23251700 & -0.30875900 & -0.63568700\end{array}$

\section{XIII}

$\begin{array}{lrrr}\text { E= }-1749.543501 \mathrm{H} & & \\ \mathrm{Cr} & -1.46182200 & -0.50456200 & 0.20538400 \\ \mathrm{C} & -2.32231400 & 0.56787400 & -1.08020400 \\ \mathrm{C} & -2.28654000 & 0.40974900 & 1.63985100 \\ \mathrm{C} & -2.92300400 & -1.60568600 & 0.25991100 \\ \mathrm{C} & -0.53285600 & -1.66853500 & 1.38564100 \\ \mathrm{C} & -0.81872300 & -1.59521100 & -1.20463000 \\ \mathrm{O} & -0.01103900 & -2.44909300 & 2.05861500 \\ \mathrm{O} & -2.90566600 & 0.85331400 & 2.51427400 \\ \mathrm{O} & -2.88918500 & 1.16144500 & -1.89959800 \\ \mathrm{O} & -0.52614700 & -2.33146800 & -2.04614800 \\ \mathrm{C} & 0.41715400 & 0.91396700 & 0.13731200 \\ \mathrm{C} & 1.37795300 & 0.60689000 & 1.26910100 \\ \mathrm{C} & 2.69889700 & 0.20762100 & 1.01617100 \\ \mathrm{C} & 0.98667600 & 0.68764500 & 2.61646600 \\ \mathrm{C} & 3.57920400 & -0.10362500 & 2.05179600 \\ \mathrm{H} & 3.04810900 & 0.12757200 & -0.01287900 \\ \mathrm{C} & 1.86611300 & 0.39879200 & 3.65273300 \\ \mathrm{H} & -0.04298200 & 0.92843100 & 2.87321600 \\ \mathrm{C} & 3.17259800 & -0.00328300 & 3.37818500 \\ \mathrm{H} & 4.59338500 & -0.42233800 & 1.81212800 \\ \mathrm{H} & 1.51815300 & 0.46489900 & 4.68310000 \\ \mathrm{H} & 3.85904800 & -0.24397600 & 4.18864800 \\ \mathrm{C} & 0.96679400 & 0.60439700 & -1.15076000 \\ \mathrm{C} & 1.73782400 & 0.10047900 & -3.62535900 \\ \mathrm{C} & 0.75304000 & 0.22707400 & -4.61991400 \\ \mathrm{C} & 3.03395900 & -0.28202300 & -4.01207500 \\ \mathrm{C} & 1.06091400 & -0.01070700 & -5.95447400 \\ \mathrm{H} & -0.26339800 & 0.50122100 & -4.33105300 \\ & & & \end{array}$

$\begin{array}{lll}3.32425000 & -0.52999800 & -5.34602400\end{array}$

$\begin{array}{lll}3.80736400 & -0.38818000 & -3.25145800\end{array}$

$\begin{array}{lll}2.34807000 & -0.39430100 & -6.34196700\end{array}$

$\begin{array}{llll}0.28272700 & 0.09245300 & -6.71219300\end{array}$

$\begin{array}{lll}4.33374600 & -0.83340900 & -5.62857300\end{array}$

$\begin{array}{lll}-3.85975400 & -2.29971800 & 0.29553500\end{array}$

$\begin{array}{lll}1.40335300 & 0.36877900 & -2.26667400\end{array}$

$\begin{array}{lll}0.07294900 & 2.48513200 & 0.16452000\end{array}$

$\begin{array}{lll}-0.73028400 & 3.03577200 & 1.28883600\end{array}$

$\begin{array}{lll}-0.56222400 & 3.14708200 & -0.98765200\end{array}$

$\begin{array}{lll}1.08461200 & 2.95567500 & 0.17406500\end{array}$

$\begin{array}{lll}-1.68252500 & 3.81536000 & 0.78467500\end{array}$

$\begin{array}{lll}-0.56285200 & 2.85208000 & 2.34173800\end{array}$

$\begin{array}{lll}-1.56635100 & 3.87966800 & -0.61168300\end{array}$

$\begin{array}{lll}-0.16204400 & 3.20420900 & -2.38502600\end{array}$

$\begin{array}{lll}-2.49021800 & 4.40830300 & 1.19295100\end{array}$

$\begin{array}{llll}0.78652400 & 2.71932400 & -2.64317500\end{array}$

$\begin{array}{lll}-0.91048900 & 3.80867300 & -3.22199700\end{array}$

$\begin{array}{lll}-0.48119900 & 3.85133000 & -4.59815300\end{array}$

$\begin{array}{llll}0.49818000 & 3.36010900 & -4.74491800\end{array}$

$\begin{array}{llll}-1.23291000 & 3.28759100 & -5.17676900\end{array}$

$\begin{array}{llll}-0.45544300 & 5.28912600 & -5.10283100\end{array}$

$\begin{array}{lll}-1.44452300 & 5.74486100 & -4.93866500\end{array}$

$\begin{array}{llll}0.26340200 & 5.86936300 & -4.50190000\end{array}$

$\begin{array}{lll}-0.08154600 & 5.36426500 & -6.57556800\end{array}$

$\begin{array}{llll}0.89723400 & 4.88242300 & -6.73097500\end{array}$

$\begin{array}{lll}-0.80803400 & 4.78049800 & -7.16348400\end{array}$

$\begin{array}{lll}-0.03637200 & 6.79529500 & -7.08358600\end{array}$

$\begin{array}{llll}0.22367800 & 6.83845800 & -8.14936100\end{array}$

$\begin{array}{lll}-1.00941100 & 7.29214200 & -6.95824200\end{array}$

$\begin{array}{lll}0.71016600 & 7.38802600 & -6.53540100\end{array}$

$\begin{array}{lll}2.68511000 & -0.65429300 & -7.77797600\end{array}$

$\begin{array}{llll}3.40841800 & 0.08038300 & -8.16009800\end{array}$

$3.14090900-1.64532900-7.90987000$

$\begin{array}{lll}1.79346000 & -0.60660900 & -8.41505900\end{array}$

\section{TS III’}

$\begin{array}{lrrr}\mathrm{E}=-1749.557501 \mathrm{H} & & \\ \mathrm{Cr} & -1.38096600 & 0.91588800 & -2.77781200 \\ \mathrm{C} & -2.51062700 & 1.21690600 & -1.28588200 \\ \mathrm{C} & -1.05741900 & -0.87049200 & -2.23119700 \\ \mathrm{C} & -2.86536400 & 0.32257600 & -3.68935600 \\ \mathrm{C} & -0.36903000 & 0.60939800 & -4.34764300 \\ \mathrm{C} & -1.77852500 & 2.67540000 & -3.34379500 \\ \mathrm{O} & 0.18680400 & 0.39682500 & -5.33988400 \\ \mathrm{O} & -0.92298900 & -1.98773900 & -1.96594700 \\ \mathrm{O} & -3.31663900 & 1.38732100 & -0.47363200 \\ \mathrm{O} & -2.10598000 & 3.71723100 & -3.72740800 \\ \mathrm{C} & 0.58024200 & 1.63401900 & -0.36418800 \\ \mathrm{C} & 0.05774200 & 0.69696300 & 0.66197300 \\ \mathrm{C} & -0.87798700 & 1.09562500 & 1.62282200 \\ \mathrm{C} & 0.51301700 & -0.62520200 & 0.66606300 \\ \mathrm{C} & -1.36261100 & 0.18383200 & 2.55444300 \\ \mathrm{H} & -1.24046800 & 2.12361500 & 1.63025600 \\ \mathrm{C} & 0.03344800 & -1.53804700 & 1.60051800 \\ \mathrm{H} & 1.25542100 & -0.93437700 & -0.07067000 \\ \mathrm{C} & -0.90805600 & -1.13462100 & 2.54516500 \\ \mathrm{H} & -2.10118500 & 0.50310400 & 3.28849000 \\ \mathrm{H} & 0.39403700 & -2.56563400 & 1.58980600\end{array}$




\begin{tabular}{cccc} 
H & -1.28790500 & -1.84720900 & 3.27621500 \\
$\mathrm{C}$ & 0.42124500 & 1.58218000 & -1.75415600 \\
$\mathrm{C}$ & 2.74625200 & 1.50240500 & -2.72537400 \\
$\mathrm{C}$ & 2.82494700 & 0.10158900 & -2.66642200 \\
$\mathrm{C}$ & 3.94336200 & 2.22221900 & -2.90824500 \\
$\mathrm{C}$ & 4.04850100 & -0.55090000 & -2.77044400 \\
$\mathrm{H}$ & 1.90964200 & -0.48080100 & -2.56376300 \\
$\mathrm{C}$ & 5.15597100 & 1.56261900 & -3.02866700 \\
$\mathrm{H}$ & 3.91995100 & 3.31205700 & -2.91263500 \\
$\mathrm{C}$ & 5.23317100 & 0.16351600 & -2.96181600 \\
$\mathrm{H}$ & 4.08258800 & -1.64005600 & -2.72457200 \\
$\mathrm{H}$ & 6.07306400 & 2.13948800 & -3.15923200 \\
$\mathrm{O}$ & -3.80432000 & -0.04877100 & -4.27050100 \\
$\mathrm{C}$ & 1.46105300 & 2.17065600 & -2.55061900 \\
$\mathrm{~N}$ & 1.23791600 & 3.40847000 & -3.07603800 \\
$\mathrm{C}$ & 0.69797000 & 4.31614400 & -2.19583300 \\
$\mathrm{H}$ & 0.35945300 & 5.26450100 & -2.60978700 \\
$\mathrm{C}$ & 1.57716200 & 3.81730900 & -4.44997500 \\
$\mathrm{H}$ & 2.16288500 & 2.99556900 & -4.88026100 \\
$\mathrm{H}$ & 0.63631800 & 3.86958400 & -5.02213400 \\
$\mathrm{C}$ & 2.33172500 & 5.12988100 & -4.56593100 \\
$\mathrm{H}$ & 1.66766700 & 5.97802800 & -4.33978200 \\
$\mathrm{H}$ & 3.14494300 & 5.16612300 & -3.82257800 \\
$\mathrm{C}$ & 2.90697000 & 5.30235500 & -5.96514400 \\
$\mathrm{H}$ & 3.63186100 & 4.49441400 & -6.15761900 \\
$\mathrm{H}$ & 2.10395600 & 5.17746600 & -6.70983800 \\
$\mathrm{C}$ & 3.57511300 & 6.65352900 & -6.14990200 \\
$\mathrm{H}$ & 4.38621400 & 6.79726700 & -5.42124000 \\
$\mathrm{H}$ & 4.00755400 & 6.75996600 & -7.15336400 \\
$\mathrm{H}$ & 2.85623500 & 7.47345800 & -6.00911000 \\
$\mathrm{C}$ & 0.76795200 & 4.17011100 & -0.82361000 \\
$\mathrm{C}$ & 0.93923400 & 3.04221900 & 0.04372600 \\
$\mathrm{O}$ & 0.68556200 & 5.30539900 & -0.04612900 \\
$\mathrm{C}$ & 1.04270200 & 3.59710500 & 1.38062500 \\
$\mathrm{H}$ & 1.81606900 & 2.06321600 & -0.17251400 \\
$\mathrm{C}$ & 0.85519000 & 4.92518300 & 1.24973700 \\
$\mathrm{H}$ & 1.18902000 & 3.05352600 & 2.30659200 \\
$\mathrm{H}$ & 0.81392100 & 5.72631800 & 1.97792400 \\
$\mathrm{C}$ & 6.55211500 & -0.53053100 & -3.1011300 \\
$\mathrm{H}$ & 6.46000200 & -1.61018900 & -2.93127400 \\
$\mathrm{H}$ & 6.97633200 & -0.38504900 & -4.10489100 \\
$\mathrm{H}$ & 7.28855900 & -0.13680400 & -2.38690200 \\
& & & \\
\hline
\end{tabular}

\begin{tabular}{lrrr}
$\mathrm{C}$ & -2.00030100 & 2.19799700 & 0.43214500 \\
$\mathrm{C}$ & -0.71652700 & 0.35069300 & 1.29549200 \\
$\mathrm{C}$ & -3.10096100 & 1.78199700 & 1.17795900 \\
$\mathrm{H}$ & -2.06587000 & 3.08965500 & -0.19451500 \\
$\mathrm{C}$ & -1.81157200 & -0.05975500 & 2.05042300 \\
$\mathrm{H}$ & 0.22233100 & -0.20520400 & 1.33923000 \\
$\mathrm{C}$ & -3.00800200 & 0.65338200 & 1.99018700 \\
$\mathrm{H}$ & -4.03270200 & 2.34468400 & 1.12892900 \\
$\mathrm{H}$ & -1.73310800 & -0.94215000 & 2.68466400 \\
$\mathrm{H}$ & -3.86676800 & 0.33197100 & 2.57831700 \\
$\mathrm{C}$ & 0.91396200 & 1.47686400 & -1.43741500 \\
$\mathrm{C}$ & 3.14448200 & 1.43280300 & -2.77356800 \\
$\mathrm{C}$ & 3.50396200 & 0.12275100 & -2.41904500 \\
$\mathrm{C}$ & 3.89904800 & 2.05664600 & -3.77418800 \\
$\mathrm{C}$ & 4.50621100 & -0.55967800 & -3.08966100 \\
$\mathrm{H}$ & 2.99890300 & -0.35173400 & -1.58041800 \\
$\mathrm{C}$ & 4.90612800 & 1.36786500 & -4.44598200 \\
$\mathrm{H}$ & 3.71779600 & 3.09468100 & -4.04774000 \\
$\mathrm{C}$ & 5.21812100 & 0.04415800 & -4.13409100 \\
$\mathrm{H}$ & 4.75585600 & -1.57883900 & -2.78939700 \\
$\mathrm{H}$ & 5.46487700 & 1.87800900 & -5.23206700 \\
$\mathrm{O}$ & -1.71484200 & -1.74585200 & -4.58898200 \\
$\mathrm{C}$ & 2.11527000 & 2.10789300 & -1.95694100 \\
$\mathrm{~N}$ & 1.94237600 & 3.53938000 & -2.22856400 \\
$\mathrm{C}$ & 2.07874600 & 4.30650600 & -1.18734400 \\
$\mathrm{H}$ & 1.87859600 & 5.37648800 & -1.21638300 \\
$\mathrm{H}$ & 5.92639200 & -1.27483400 & -4.19093700 \\
$\mathrm{H}$ & 6.91755000 & -1.43361000 & -5.57284600 \\
$\mathrm{C}$ & 1.40947100 & 4.02425700 & -3.52233000 \\
$\mathrm{H}$ & 1.51686200 & 3.19970300 & -4.23683600 \\
$\mathrm{H}$ & 0.33201700 & 4.18877100 & -3.38517800 \\
$\mathrm{C}$ & 2.09197900 & 5.28482700 & -4.02130400 \\
$\mathrm{H}$ & 1.82598800 & 6.14197600 & -3.38296400 \\
$\mathrm{H}$ & 3.18630000 & 5.17197500 & -3.95436600 \\
$\mathrm{C}$ & 1.69161800 & 5.57970400 & -5.46034400 \\
$\mathrm{H}$ & 1.99087500 & 4.73144200 & -6.09703300 \\
$\mathrm{H}$ & 0.59410300 & 5.64432600 & -5.53024100 \\
$\mathrm{C}$ & 2.32053900 & 6.86177100 & -5.97603200 \\
$\mathrm{H}$ & 3.41815200 & 6.80862400 & -5.93686000 \\
$\mathrm{H}$ & 2.03580300 & 7.06117400 & -7.01708600 \\
$\mathrm{H}$ & 2.00998800 & 7.72843200 & -5.37488000 \\
$\mathrm{C}$ & 2.36586800 & 3.52774100 & 0.00216200 \\
$\mathrm{C}$ & 1.17178300 & 2.89109500 & 0.53891600 \\
$\mathrm{C}$ & 2.99180500 & 4.21256900 & 1.04772000 \\
$\mathrm{H}$ & 1.13988700 & 3.24999500 & 1.92539800 \\
\hline & 2.84071800 & 2.52105400 & -0.67006800 \\
$\mathrm{H}$ & 2.25799000 & 3.97505000 & 2.16409400 \\
$\mathrm{H}$ & 2.67158900 & 4.37184300 & 2.66421900 \\
$\mathrm{H}$ & 6.28150200 & -0.70660400 & -4.87465200 \\
$\mathrm{H}$ & & &
\end{tabular}

\section{TS IV'}

$\begin{array}{lrrr}\mathrm{E}=-1749.590572 \mathrm{H} & & \\ \mathrm{Cr} & -0.21987400 & 0.03440600 & -2.69202800 \\ \mathrm{C} & -1.70053000 & -0.36480300 & -1.59127300 \\ \mathrm{C} & 0.70628000 & -1.37475900 & -1.83937600 \\ \mathrm{C} & -1.13886300 & -1.05362500 & -3.84784500 \\ \mathrm{C} & 0.94655300 & 0.12626100 & -4.18554900 \\ \mathrm{C} & -1.11192800 & 1.61387400 & -3.18859100 \\ \mathrm{O} & 1.47797500 & 0.06832500 & -5.21466000 \\ \mathrm{O} & 1.22366900 & -2.24415000 & -1.27599300 \\ \mathrm{O} & -2.69574400 & -0.74238400 & -1.13060100 \\ \mathrm{O} & -1.68624100 & 2.59017700 & -3.43728900 \\ \mathrm{C} & 0.41170800 & 1.98865100 & -0.22259900 \\ \mathrm{C} & -0.80244600 & 1.48016700 & 0.47444400\end{array}$




\section{NMR Spectra}

$N$-butyl-6-phenyl-4- $p$-tolylbenzofuran-7-amine (5g)

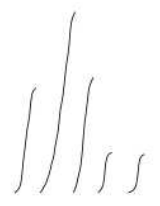<smiles>Cc1ccc(-c2cc(-c3ccccc3)c3ccoc3c2Br)cc1</smiles><smiles>C1CCCCCC1</smiles><smiles>CC=CC</smiles><smiles>C=CCCC</smiles>
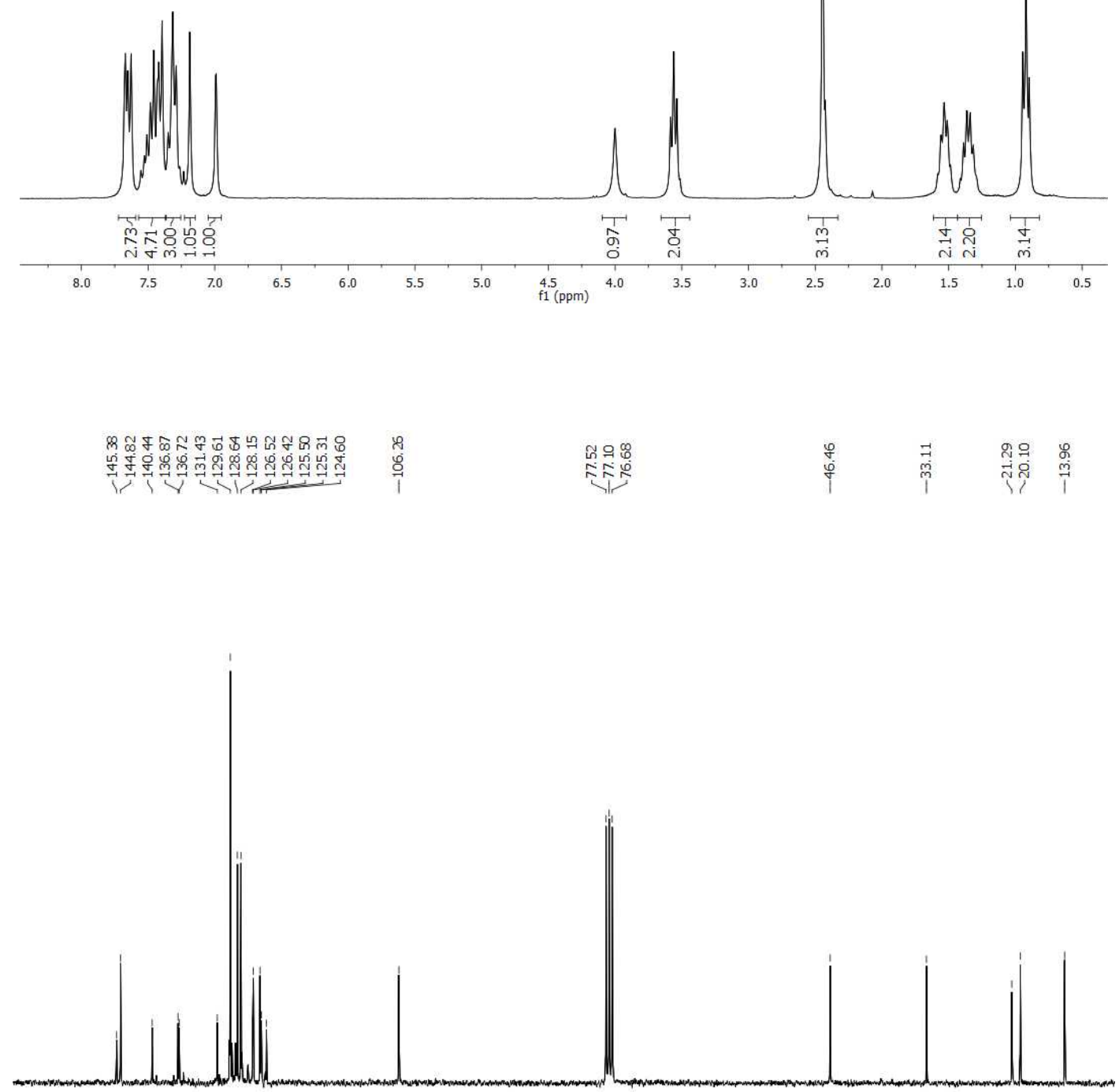

$\begin{array}{llllllllllllllllllllllllllllll}155 & 150 & 145 & 140 & 135 & 130 & 125 & 120 & 115 & 110 & 105 & 100 & 95 & 90 & \begin{array}{c}85 \\ \mathrm{f1}(\mathrm{ppm})\end{array} & 75 & 70 & 65 & 60 & 55 & 50 & 45 & 40 & 35 & 30 & 25 & 20 & 15 & 10\end{array}$ 
$N$-butyl -6-(4-methoxyphenyl)-4-phenyl benzofuran-7-amine (5i)

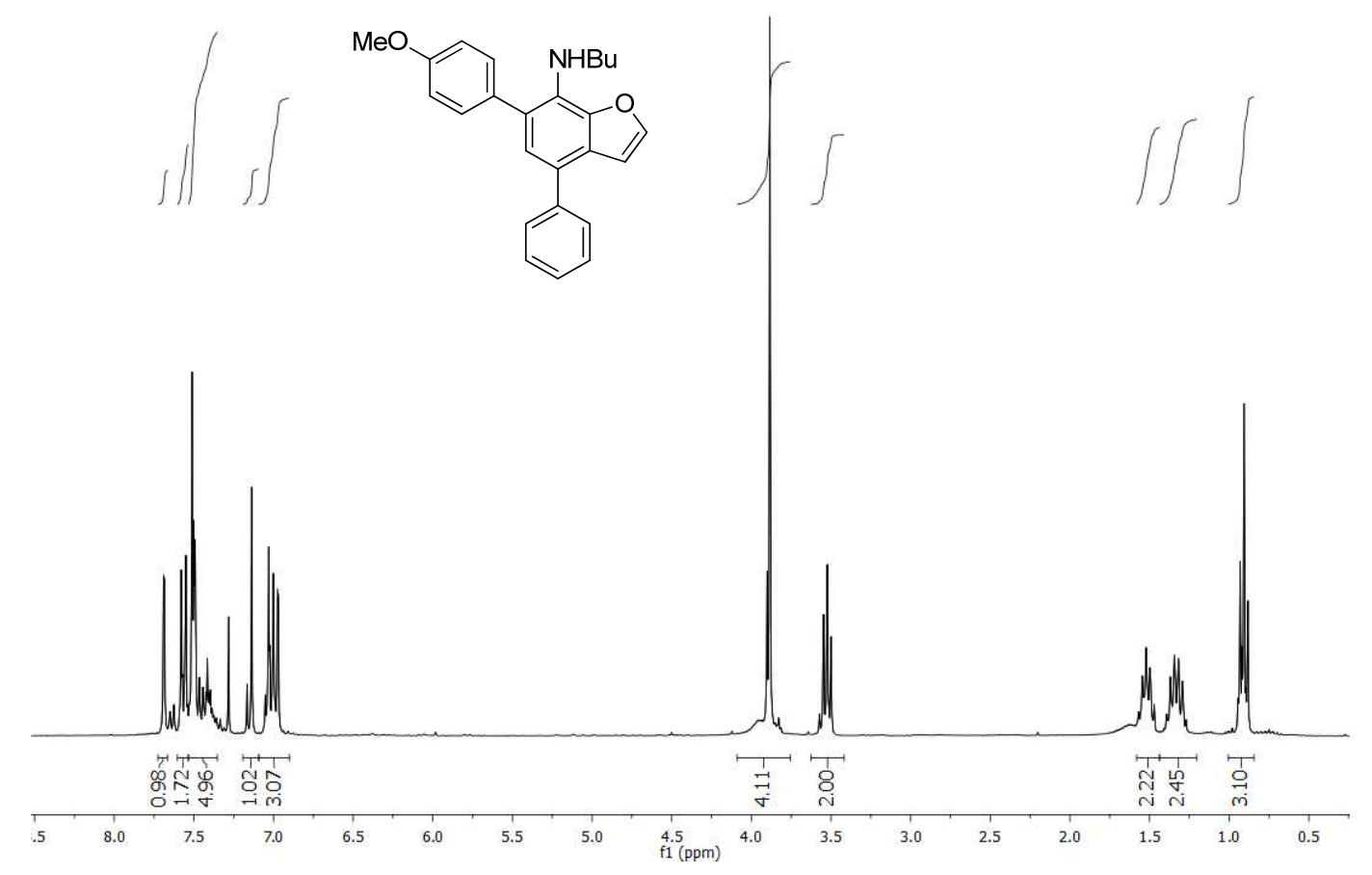

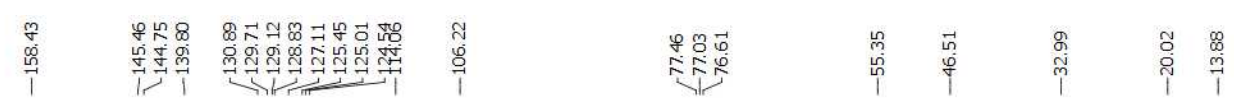

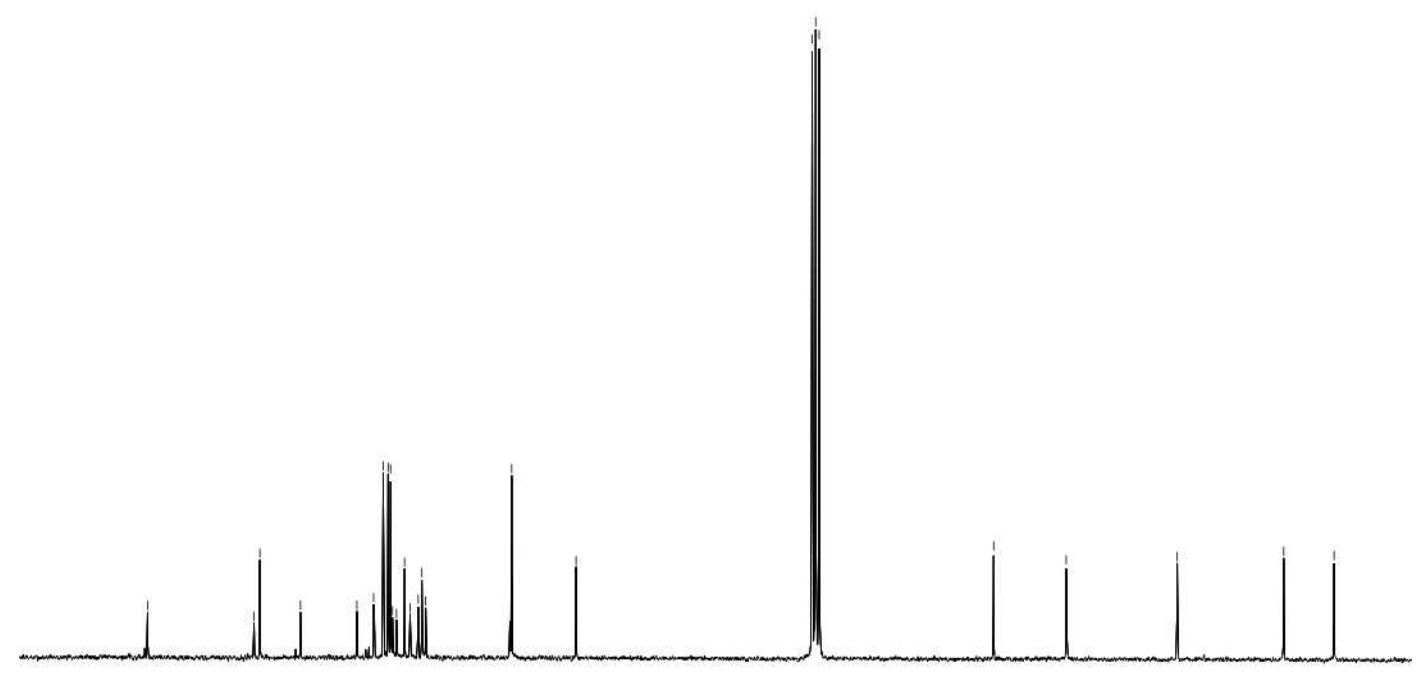

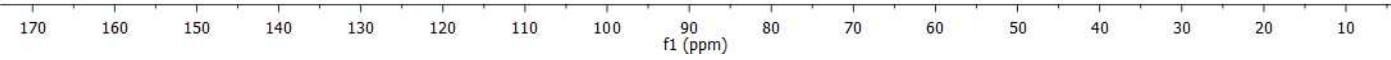


Pentacarbonyl[(1-butyl-2-furyl-4-(4-methoxyphenyl)-1,2-dihydroazet-3yl)benzylidene]chromium(0) (6i)
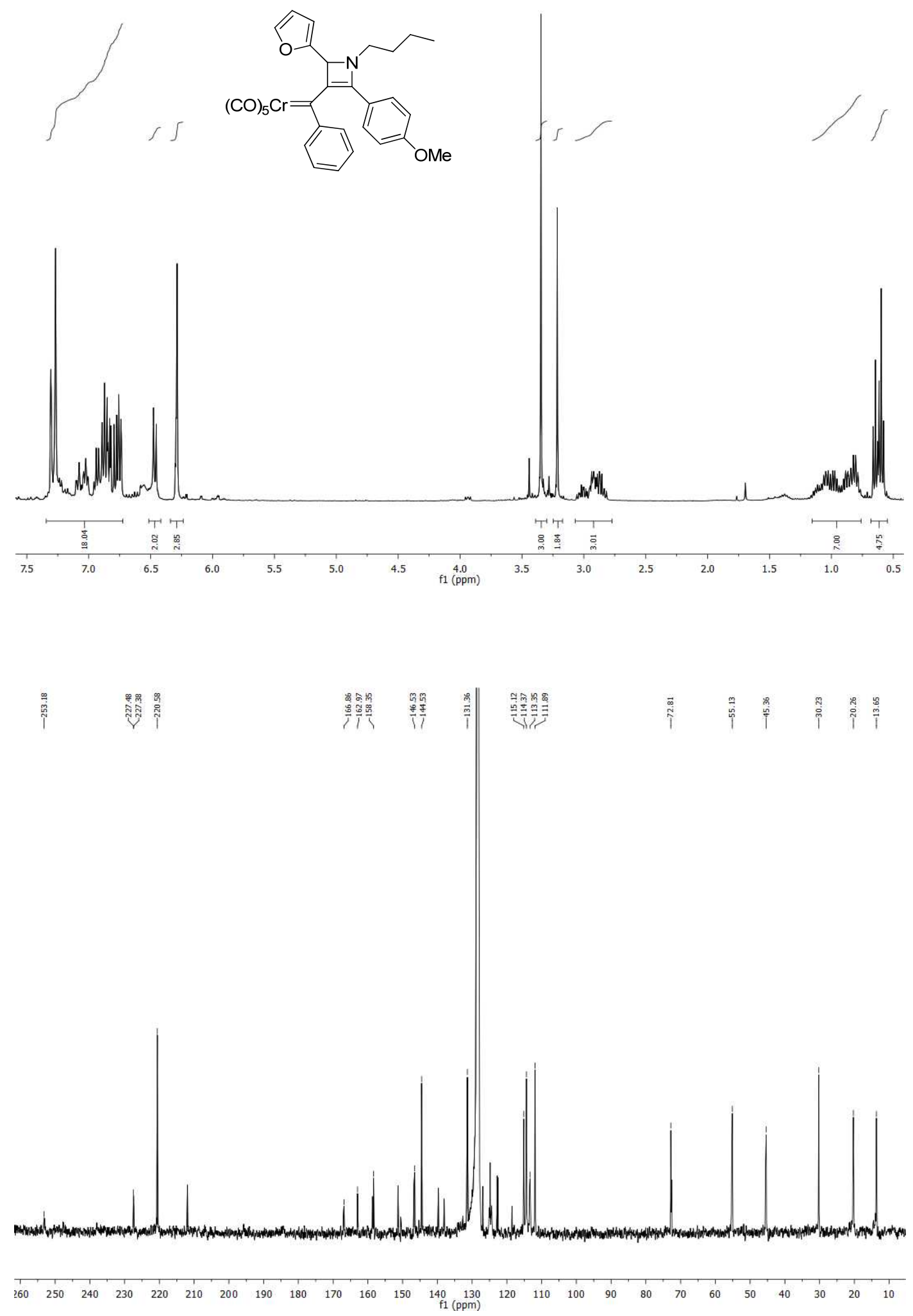
Pentacarbonyl[(1-butyl-2-furyl-4-(4-methoxyphenyl)-1,2-dihydroazet-3yl)4-methoxybenzylidene]chromium(0) (6j)
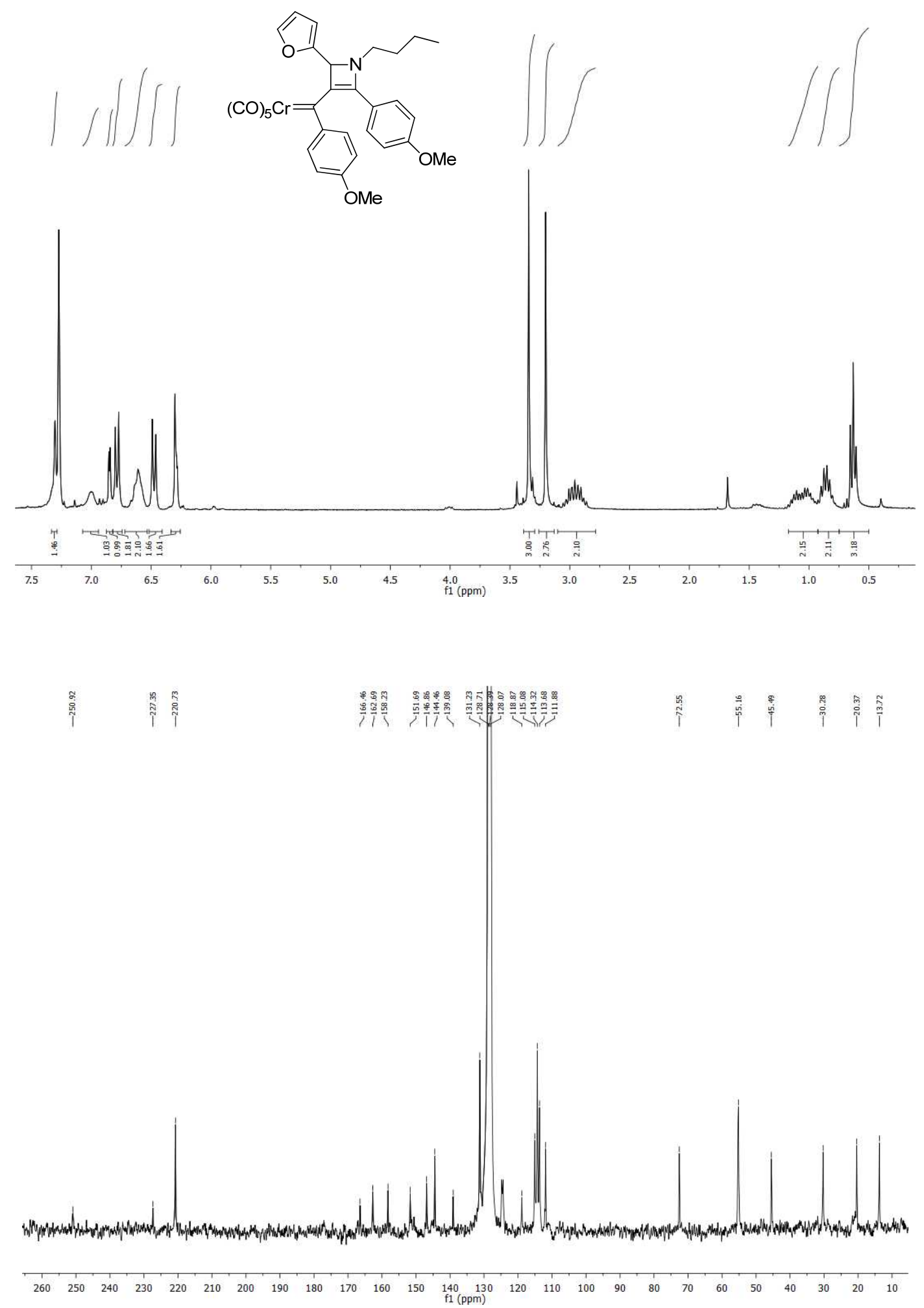